\title{
Effect of the mechanical harvest of drupes on the quality characteristics of green fermented table olives
}

\author{
Alessandra Martorana, ${ }^{a}$ Antonio Alfonzo, ${ }^{a}$ Luca Settanni,, ${ }^{a}$ Onofrio Corona, ${ }^{a}$ \\ Francesco La Croce, ${ }^{b}$ Tiziano Caruso, ${ }^{a}$ Giancarlo Moschetti ${ }^{a}$ and \\ Nicola Francesca ${ }^{a^{*}}$
}

\section{Abstract}

BACKGROUND: Because of damage caused by mechanical harvesting, the drupes for table olive production are traditionally hand harvested. Until now, no data have been available on the microbiological and chemical features of mechanically harvested drupes during fermentation.

RESULTS: Drupes mechanically harvested and inoculated with Lactobacillus pentosus OM13 were characterized by the lowest concentrations of potential spoilage microorganisms. On the other hand, drupes mechanically harvested and subjected to spontaneous fermentation showed the highest concentration of Enterobacteriaceae and pseudomonads during transformation. The lowest decrease of $\mathrm{pH}$ (4.20) was registered for the trials inoculated with the starter culture. Differences in terms of volatile organic compounds were estimated among trials. Multivariate analysis showed that the olives processed from the drupes mechanically harvested and inoculated with starter were closely related to control production (drupes manually harvested) in terms of microbiological and pH values. Sensory analysis evidenced negative evaluations only for the uninoculated trials.

CONCLUSION: Drupes mechanically harvested and subjected to a driven fermentation with Lactobacillus pentosus OM13 determined the production of table olives with appreciable organoleptic features. Thus mechanical harvesting performed using a trunk shaker equipped with an inverse umbrella and the addition of starter lactic acid bacteria represents a valuable alternative to manual harvesting for table olive production at the industrial level.

(c) 2015 Society of Chemical Industry

Supporting information may be found in the online version of this article.

Keywords: lactic acid bacteria; yeasts; Lactobacillus pentosus OM13; Nocellara del Belice table olive fermentation; mechanical harvest; manual harvest

\section{INTRODUCTION}

Drupes to be transformed into table olives have to be intact. Skin and flesh of drupes are extremely sensitive to scratches and wounds that may occur during harvesting. For this reason, only hand harvesting carried out with accuracy can avoid fruit damage. Mechanical harvesting is not generally applied to drupes to be transformed into table olives; this kind of harvesting might damage (scratches, wounds on skins) drupes, which can not be further processed. ${ }^{1,2}$ However, susceptibility to damage depends on the variety, texture, maturity stage, water content, firmness, temperature, size and shape, as well as internal fruit factors such as cell wall, strength and elasticity, cell shape and structure. ${ }^{3-5}$ Furthermore, as stated by Jiménez-Jiménez et al., ${ }^{6}$ the mechanical damage from impact is strictly related to the impact energy level and the time after impact.

The mechanical harvesting of drupes for olive oil production has been deeply studied ${ }^{7,8}$ and this practice is widespread. Conversely, knowledge of the effect of this harvest on table olives is quite limited..$^{9-11}$ Mechanical harvesting, mostly applied to drupes for table olives, is performed using large trunk-shaking or vibrating machines that operate at a well-defined vibration frequency and shaking time. ${ }^{12-14}$ Recently, Gambella et al. ${ }^{15}$ tested three coating materials (silicone, vulcanized rubber and natural rubber) with different thicknesses and rotational speeds to reduce the percentage of damaged fruits.

Olive drupes cannot be eaten unprocessed because of the presence of oleuropein, which is a bitter glucoside consisting of glucose, elenolic acid and o-diphenol hydroxytyrosol compounds. ${ }^{16}$ A variety of technological methods are commonly applied to produce table olives. ${ }^{17,18}$ The 'Greek' processing style, also known as

\footnotetext{
Correspondence to: Nicola Francesca, Dipartimento Scienze Agrarie e Forestali, Università degli Studi di Palermo, Viale delle Scienze 4, 90128 Palermo, Italy. E-mail: nicola.francesca@unipa.it

a Dipartimento Scienze Agrarie e Forestali, Università degli Studi di Palermo, Viale delle Scienze 4, 90128 Palermo, Italy

b Geolive Belice SAS, Castelvetrano, Trapani, Italy
} 
the 'natural method', is a common method employed in Mediterranean countries, ${ }^{19}$ even though productions carried out at an industrial level rely on starter lactic acid bacteria (LAB), often Lactobacillus plantarum and/or Lactobacillus pentosus. ${ }^{16,17,20,21}$ Generally, the direct inoculation of commercial starter cultures into olive brine ensures a rapid increase of LAB populations, with a sudden decrease in $\mathrm{pH}$. This procedure limits the risk of off-flavour generation due to growth of spoilage microrganisms. ${ }^{16,22-24}$ For this reason, the use of selected strains is becoming a common practice for table olive fermentation. ${ }^{24-27}$ The International Olive Council (IOC) estimated a total world production of 2.5 million tons of olives for the 2012/2013 campaign, with 76000 tons located in Italy. ${ }^{28}$

Sicily (southern Italy) is the most important Italian region for table olive production. ${ }^{29}$ A consistent part of this production is based on Nocellara del Belice cultivar and the resulting product enjoys the status of Protected Denomination of Origin (PDO) obtained through Regulation EC No. 134/1998. Traditionally, the harvesting of Nocellara del Belice drupes is time consuming because it is performed manually. The present research was aimed at studying the applicability of a trunk shaker machine equipped with an inverted umbrella catching the detached, falling fruits of Nocellara del Belice to reduce the number of damaged drupes, as well as to limit the extent of damage and to evaluate the influence of this practice on the final product. To this purpose, the microbiological, chemical and sensory parameters were monitored during table olive processing.

\section{EXPERIMENTAL}

\section{Experimental table olive production and sample collection}

Table olive transformation was carried out on drupes of the cultivar Nocellara del Belice produced by olive groves (located in Castelvetrano, Trapani province, Sicily, Italy; $37^{\circ} 36^{\prime} 46^{\prime \prime} \mathrm{N} / 12^{\circ}$ $\left.50^{\prime} 52^{\prime \prime} \mathrm{E}\right)$, the main area for Nocellara del Belice PDO production. Olive drupes were mechanically harvested using a trunk shaker equipped with an inverse umbrella (model 'SICMA F3 umbrella olive harvester'; SICMA Srl, Acconia di Curinga, Catanzaro, Italy). The machine was equipped with a high-frequency and self-breaking vibrating head mounted over a telescopic arm; the reverse umbrella (7 $\mathrm{m}$ in diameter), case (containing up to $500 \mathrm{~kg}$ of drupes) and unload unit were hydraulically controlled. Samples of drupes were collected in 2012 and 2013 from the mass of fruits obtained shaking 50 trees per year.

The samples of olive drupes were transferred to two vats $(180 \mathrm{~L}$ volume). Each vat contained $150 \mathrm{~kg}$ olives and $30 \mathrm{~L}$ brine composed of $\mathrm{NaCl} 9 \%(\mathrm{w} / \mathrm{v})$. One vat was inoculated with $0.15 \mathrm{~g} \mathrm{~kg}^{-1}$ of the freeze-dried autochthonous strain L. pentosus OM13, already tested for Nocellara del Belice table olives, ${ }^{22}$ and represented the trial mechanical $A$ (MCA). The other vat was uninoculated and represented the trial mechanical $B(M C B)$, which was spontaneously fermented. Two additional trials performed with drupes manually harvested were included in the experimental plan: manual $A$ (MNA) inoculated as reported above for the trial MCA and manual $B$ (MNB) subjected to spontaneous fermentation. The fermentation of all trials was carried out at room temperature for 210 days and periodically monitored. Samples of brine (about $50 \mathrm{~mL}$ ) were collected before starter culture inoculation, immediately after its addition and at 3, 6, 9, 15, 30, 50, 70, 90, 130,170 and 210 days of fermentation. The experiment was performed in triplicate (three vessels per trial) in two consecutive years (2012 and 2013).

\section{Physicochemical and microbiological analyses}

The $\mathrm{pH}$ values of brine samples were determined by $\mathrm{pH}$ meter (BASIC 20+; Crison Instruments SA, Barcelona, Spain). Salt concentration was routinely analysed as reported by Garrido Fernández et al. ${ }^{30}$

Decimal dilutions of brines were prepared in Ringer's solution (Sigma-Aldrich, Milan, Italy) and different microbial groups, such as mesophilic rod LAB, yeasts, Enterobacteriaceae, pseudomonads, staphylococci and coagulase-positive staphylococci (CPS), enumerated as reported by Martorana et al. ${ }^{31}$ Analyses were performed in triplicate. All media and supplements used were supplied from Oxoid (Thermofisher, Basingstoke, UK).

\section{Isolation and phenotypic grouping of LAB}

Presumptive LAB (at least four colonies with the same colour, morphology, margin, surface and elevation) were collected from the highest plated dilutions following their growth on MRS agar. The isolates were purified by successive subculturing and the purity of the isolates was checked microscopically. Gram-positive (Gregersen $\mathrm{KOH}$ method) and catalase-negative isolates (determined in presence of $\mathrm{H}_{2} \mathrm{O}_{2} 5 \%, \mathrm{v} / \mathrm{v}$ ) were stored in broth containing $20 \%(\mathrm{v} / \mathrm{v})$ glycerol at $-80^{\circ} \mathrm{C}$ pending further experimentation.

LAB were initially subjected to a phenotypic grouping based on cell morphology and disposition, determined by an optical microscope, growth at 15 and $45^{\circ} \mathrm{C}$ and metabolism type, testing the ability to produce $\mathrm{CO}_{2}$ from glucose. The last assay was carried out with the same growth medium used for isolation, without citrate, from which certain $L A B$ can result in gas formation. Obligate homofermentative metabolism was determined by the absence of growth in the presence of a mixture of pentose carbohydrates (xylose, arabinose and ribose; $8 \mathrm{~g} \mathrm{~L}^{-1}$ each) in place of glucose.

\section{Genotypic investigation of LAB at strain and species level}

DNA from $L A B$ isolates was extracted using the InstaGene Matrix kit (Bio-Rad Laboratories, Hercules, CA, USA) according to the manufacturer's instructions. Strain differentiation was performed by random amplification of polymorphic DNA-PCR (RAPD-PCR) analysis in a $25 \mu \mathrm{L}$ reaction mix using single primers M13, AB111 and AB106 as previously described by Settanni et al. ${ }^{32}$ The identification at species level was performed by multiplex PCR analysis based on the recA gene with species-specific primers for Lactobacillus pentosus, L. plantarum and L. paraplantarum, as described by Torriani et al. ${ }^{33}$

One representative culture for each multiplex cluster and all strains that did not show amplification by multiplex PCR analysis were analysed by $16 \mathrm{~S}$ rRNA gene sequencing as described by Weisburg et al. ${ }^{34}$ DNA sequencing reactions were performed at PrimmBiotech Srl (Milan, Italy). The identities of the sequences were determined by BlastN search against the NCBI non-redundant sequence database, located at http://www.ncbi.nlm.nih.gov, and those of the sole type strains within the database EZTaxon, located at http://www.ezbiocloud.net/eztaxon.

\section{Isolation, grouping and identification of yeasts}

Yeasts were collected from DRBC medium. At least five colonies per morphology were randomly collected from the agar plates, purified to homogeneity after several subculturing steps onto DRBC medium and subjected to genetic characterization.

DNA extraction was performed as reported above. All selected isolates were preliminarily grouped by restriction fragment length polymorphism (RFLP) analysis of the region spanning the internal 
transcribed spacers (ITS1 and ITS2) and the 5.8S rRNA gene as reported by Esteve-Zarzoso et al. ${ }^{35}$ One isolate per group was identified at species level by sequencing the D1/D2 region of the $26 \mathrm{~S}$ rRNA gene to confirm the preliminary identification obtained by RFLP analysis. The D1/D2 region was amplified and polymerase chain reaction (PCR) products were visualized as described by Settanni et l. $^{36}$ The reaction of DNA sequencing and the identities of sequences were determined as reported above.

\section{Volatile organic compounds (VOCs)}

Volatile compounds were determined at 210 days of fermentation by solid phase micro-extraction technique in head space followed by gas chromatography-mass spectrometry (HS-SPMEGC-MS). ${ }^{25,37}$ Drupes were homogenized and transferred $(0.50 \mathrm{~g})$ into $2 \mathrm{~mL}$ vials with pierceable silicone rubber septa coated with polytetrafluoroethylene (PTFE) film. Fifty microliters of 2-pentanol-4-methyl methanol solution $\left(0.981 \mu \mathrm{g} \mathrm{mL}^{-1}\right)$ were used as internal standard. A Supelco SPME (Bellefonte, PA) holder and fibre was coated with divinylbenzene-carboxenpolydimethylsiloxane. The vials were heated at a controlled temperature $\left(40 \pm 0.5^{\circ} \mathrm{C}\right)$ in order to reach equilibrium and $30 \mathrm{~min}$ exposure time. The GC-MS conditions were used as described by Corona..$^{38}$ Collected data were processed with the instrument data system. Olive volatile compounds were identified by comparison of the retention times with those of the reference compounds (NIST/EPA/MSDC Mass Spectral Database, TG House, Cambridge, UK). Semi-quantitative determination was carried out by the internal standard method. The calibration curve was constructed with readings on five 2-pentanol-4-methyl methanol solutions with concentrations ranging from 1.5 to $8 \mu \mathrm{g} \mathrm{mL}^{-1}\left(R^{2}=0.994\right)$. All analyses were performed in triplicate.

\section{Sensory evaluation}

Evaluation of the sensory profiles of the experimental olives was performed using a descriptive method (UNI 10957, 2003) ${ }^{39}$ as reported by Aponte et al. ${ }^{22}$ The analysis was applied to olives at the end of fermentation (day 210).

Twelve judges (six females and six males, $22-35$ years old) were trained in preliminary sessions using different samples of commercial table olives of the cultivar Nocellara del Belice, in order to develop a common vocabulary for the description of the sensory attributes of the experimental samples and to familiarize with scales and procedures. Each attribute was extensively described and explained to avoid any doubt about the relevant meaning. The sensory attributes cited with a frequency higher than $60 \%$ by panellists were selected for sample evaluation. Thus 15 descriptors were included in the analysis for the external aspect (green colour intensity), odour (green olive aroma, complexity and off-odours), taste (crispness, easy stone detachment from the flesh, juicy, sweet, sour, bitter, salt, astringent and complexity) and off-flavours. The olive samples were randomly evaluated by assigning a score between 1.00 (absence of sensation) and 9.00 (extremely intense) in individual booths under incandescent white light.

\section{Statistical and explorative multivariate analysis}

Data of $\mathrm{pH}$, microbiological investigation and sensory evaluation were analysed using a generalized linear model (GLM) that included the effects of samples; Student's $t$-test was used for mean comparison. Post hoc Tukey's method was applied for pairwise comparison. Statistical significance was attributed to $P$-values of
$<0.05$. The resulting scores from the sensory analysis were averaged and compared. The analysis of variance (ANOVA) test was applied to identify significant differences among olive attributes. Combined ANOVA of pH values and microbiological counts was performed on data collected over 2 years of experimentation. All parameters were analysed by two-way ANOVA within each year. Simple effects tests were used to examine significant two-way interactions and the least significant difference (LSD) procedure was used for pairwise comparison.

In addition, explorative multivariate analysis was employed to investigate the relationship among data obtained from the different experimentations. A hierarchical cluster analysis (HCA) (joining, tree clustering) was carried out for grouping the trials according to their similarity, measured by Euclidean distances, whereas cluster aggregation was based on the single linkage method..$^{40}$ HCA is a graphical representation of a matrix of distances such as the dendrogram where the objects (strains) are joined together in a hierarchical ascendant analysis from the closest one, i.e. the most similar, to the furthest apart, which is the most different.

Furthermore, the principal component analysis (PCA) was employed to investigate the relationships among samples. The input matrix used for HCA and PCA consisted of the total area under growth/decline curves of $L A B$, yeasts, enterobacteria, pseudomonads, staphylococci, CPS and $\mathrm{pH}$ values. ${ }^{41,42}$ Areas were calculated by integration using OriginPro 7.5 software (OriginLab Corporation, Northampton, MA, USA). In addition, other relevant indexes of $\mathrm{pH}$ and microbial changes were taken into account as follows: maximum and minimum values of $\mathrm{pH}$; maximum and minimum values of microbial populations. ${ }^{41}$

PCA was also employed to investigate the relationship among olive samples based on VOC profile, as well as sensory analysis. ${ }^{43,44}$

The number of principal factors was selected according to the Kaiser criterion ${ }^{45}$ and only factors with eigenvalues higher than 1.00 were retained. All data were preliminarily evaluated using Barlett's sphericity test, ${ }^{46,47}$ in order to check the statistically significant difference among samples within each dataset.

Statistical data processing and graphical construction were achieved using the SPSS software package (SPSS Inc., Chicago, IL, USA), STATISTICA software version 10 (StatSoft Inc., Tulsa, OK, USA) and XLStat software version 7.5.2 (Addinsoft, New York, NY, USA) for Excel.

\section{RESULTS}

\section{pH values and microbiological loads}

The results of $\mathrm{pH}$ measurements and microbial analyses of the brines collected during the entire process of table olive production are reported in Table 1.

The $\mathrm{pH}$ value was 7.3 at the beginning of the transformation (day 0 ) and 4.2 at the end of the process (day 210) for all trials in both years of research. Trial MCA showed the highest decrease of $\mathrm{pH}$ at day 6 of fermentation and reached $\mathrm{pH}$ values significantly lower than MCB at day 30 . From day 50 onwards, the pH decreased approximately to 4.2 for all treatments. However, the most rapid decrease of $\mathrm{pH}$ was registered for MCA and MNA.

The microbial groups mostly represented on untreated olives mechanically harvested were yeasts (almost $5.0 \log \mathrm{CFU} \mathrm{g}{ }^{-1}$ in both years) and LAB (around $2.0 \log$ CFU g ${ }^{-1}$ in both years). The concentrations of Enterobacteriaceae and pseudomonads were comparable to that of $L A B$. Yeasts and $L A B$ were more represented than other microbial groups even when manually harvested olives 
Table 1. Values of $\mathrm{pH}$ and microbial concentrations of samples collected during 2012 and 2013 table olive production

\begin{tabular}{|c|c|c|c|c|c|c|c|c|c|c|c|c|}
\hline & \multicolumn{12}{|c|}{ Days of fermentation } \\
\hline & 0 & 3 & 6 & 9 & 15 & 30 & 50 & 70 & 90 & 130 & 170 & 210 \\
\hline \multicolumn{13}{|l|}{ pH 2012} \\
\hline Trial MCA & $7.2 \pm 0.3 a$ & $5.2 \pm 0.1 a$ & $4.0 \pm 0.2 a$ & $4.0 \pm 0.2 a$ & $3.8 \pm 0.1 a$ & $3.7 \pm 0.0 \mathrm{a}$ & $3.8 \pm 0.1 a$ & $3.7 \pm 0.2 \mathrm{a}$ & $4.0 \pm 0.1 a$ & $4.4 \pm 0.2 a$ & $4.3 \pm 0.1 a$ & $4.2 \pm 0.2 a$ \\
\hline Trial MCB & $7.3 \pm 0.1 \mathrm{a}$ & $5.7 \pm 0.1 b$ & $5.4 \pm 0.2 b$ & $5.3 \pm 0.1 b$ & $4.2 \pm 0.0 a$ & $4.2 \pm 0.1 \mathrm{a}$ & $4.3 \pm 0.1 b$ & $4.3 \pm 0.2 a$ & $4.1 \pm 0.3 a$ & $4.4 \pm 0.1 \mathrm{a}$ & $4.5 \pm 0.3 a$ & $4.3 \pm 0.2 a$ \\
\hline Trial MNA & $7.3 \pm 0.2 a$ & $5.2 \pm 0.2 a$ & $4.3 \pm 0.3 a$ & $4.1 \pm 0.2 \mathrm{a}$ & $3.7 \pm 0.2 \mathrm{a}$ & $3.9 \pm 0.2 \mathrm{a}$ & $3.7 \pm 0.2 a$ & $3.9 \pm 0.2 a$ & $4.1 \pm 0.2 a$ & $4.3 \pm 0.1 a$ & $4.2 \pm 0.1 \mathrm{a}$ & $4.0 \pm 0.1 a$ \\
\hline Trial MNB & $7.2 \pm 0.2 \mathrm{a}$ & $6.6 \pm 0.1 c$ & $6.3 \pm 0.2 c$ & $5.7 \pm 0.2 b$ & $4.9 \pm 0.2 b$ & $4.3 \pm 0.2 \mathrm{a}$ & $4.4 \pm 0.2 b$ & $4.1 \pm 0.3 a$ & $4.1 \pm 0.2 \mathrm{a}$ & $4.3 \pm 0.2 a$ & $4.7 \pm 0.1 \mathrm{a}$ & $4.4 \pm 0.1 a$ \\
\hline \multicolumn{13}{|l|}{ pH 2013} \\
\hline Trial MCA & $7.3 \pm 0.1 \mathrm{a}$ & $5.0 \pm 0.2 a$ & $4.9 \pm 0.3 a$ & $4.4 \pm 0.2 a$ & $4.2 \pm 0.1 a$ & $3.9 \pm 0.1 \mathrm{a}$ & $4.1 \pm 0.1 \mathrm{a}$ & $4.3 \pm 0.1 a$ & $4.1 \pm 0.2 a$ & $4.0 \pm 0.2 a$ & $4.1 \pm 0.2 \mathrm{a}$ & $4.1 \pm 0.1 \mathrm{a}$ \\
\hline Trial MCB & $7.3 \pm 0.1 \mathrm{a}$ & $6.1 \pm 0.3 b$ & $6.0 \pm 0.2 b$ & $5.3 \pm 0.3 b$ & $4.9 \pm 0.1 b$ & $4.6 \pm 0.1 \mathrm{a}$ & $4.2 \pm 0.2 a$ & $4.3 \pm 0.2 a$ & $4.4 \pm 0.3 a$ & $4.3 \pm 0.1 \mathrm{a}$ & $4.4 \pm 0.2 \mathrm{a}$ & $4.3 \pm 0.2 a$ \\
\hline Trial MNA & $7.4 \pm 0.2 a$ & $5.2 \pm 0.2 a$ & $4.9 \pm 0.3 a$ & $4.4 \pm 0.1 a$ & $4.1 \pm 0.2 a$ & $4.1 \pm 0.2 \mathrm{a}$ & $4.2 \pm 0.3 a$ & $4.0 \pm 0.1 a$ & $4.2 \pm 0.5 a$ & $4.0 \pm 0.4 a$ & $4.2 \pm 0.2 a$ & $4.0 \pm 0.3 a$ \\
\hline Trial MNB & $7.1 \pm 0.1 \mathrm{a}$ & $5.3 \pm 0.2 a$ & $5.6 \pm 0.1 b$ & $5.3 \pm 0.2 b$ & $4.7 \pm 0.4 b$ & $4.5 \pm 0.3 a$ & $4.4 \pm 0.3 a$ & $4.4 \pm 0.2 \mathrm{a}$ & $4.0 \pm 0.2 \mathrm{a}$ & $4.1 \pm 0.3 a$ & $4.1 \pm 0.1 \mathrm{a}$ & $4.1 \pm 0.1 \mathrm{a}$ \\
\hline \multicolumn{13}{|l|}{ MRS 2012} \\
\hline Trial MCA & $7.0 \pm 0.2 b$ & $6.2 \pm 0.1 c$ & $7.3 \pm 0.0 b$ & $6.9 \pm 0.3 b$ & $7.0 \pm 0.1 b$ & $7.3 \pm 0.1 b$ & $6.9 \pm 0.3 b$ & $6.8 \pm 0.1 c$ & $5.9 \pm 0.3 b$ & $5.7 \pm 0.2 a$ & $5.8 \pm 0.1 b$ & $5.2 \pm 0.1 a$ \\
\hline Trial MCB & $1.0 \pm 0.2 \mathrm{a}$ & $4.7 \pm 0.1 b$ & $4.5 \pm 0.2 a$ & $4.4 \pm 0.1 a$ & $5.1 \pm 0.2 a$ & $5.6 \pm 0.3 a$ & $5.1 \pm 0.1 a$ & $5.2 \pm 0.2 a$ & $4.8 \pm 0.2 a$ & $5.1 \pm 0.3 a$ & $4.7 \pm 0.1 \mathrm{a}$ & $5.0 \pm 0.2 a$ \\
\hline Trial MNA & $7.0 \pm 0.4 b$ & $6.7 \pm 0.2 d$ & $6.9 \pm 0.4 b$ & $7.2 \pm 0.1 b$ & $7.1 \pm 0.4 b$ & $7.0 \pm 0.3 b$ & $7.4 \pm 0.1 c$ & $6.3 \pm 0.3 b$ & $6.0 \pm 0.1 b$ & $5.3 \pm 0.2 a$ & $5.9 \pm 0.1 b$ & $5.7 \pm 0.2 b$ \\
\hline Trial MNB & $1.1 \pm 0.1 \mathrm{a}$ & $4.0 \pm 0.1 \mathrm{a}$ & $4.2 \pm 0.1 \mathrm{a}$ & $4.6 \pm 0.0 \mathrm{a}$ & $5.3 \pm 0.1 a$ & $5.5 \pm 0.2 \mathrm{a}$ & $5.8 \pm 0.3 b$ & $5.3 \pm 0.3 a$ & $4.5 \pm 0.2 \mathrm{a}$ & $5.3 \pm 0.3 a$ & $5.0 \pm 0.2 \mathrm{a}$ & $4.8 \pm 0.3 a$ \\
\hline \multicolumn{13}{|l|}{ MRS 2013} \\
\hline Trial MCA & $7.2 \pm 0.1 b$ & $7.2 \pm 0.2 d$ & $7.1 \pm 0.0 c$ & $7.5 \pm 0.1 b$ & $7.5 \pm 0.2 c$ & $6.8 \pm 0.1 b$ & $6.9 \pm 0.1 c$ & $7.1 \pm 0.3 c$ & $6.2 \pm 0.3 c$ & $6.0 \pm 0.3 b$ & $6.1 \pm 0.1 b$ & $6.0 \pm 0.2 b$ \\
\hline Trial MCB & $1.1 \pm 0.2 \mathrm{a}$ & $4.7 \pm 0.3 b$ & $4.5 \pm 0.1 \mathrm{a}$ & $4.4 \pm 0.2 a$ & $4.7 \pm 0.3 a$ & $5.6 \pm 0.1 \mathrm{a}$ & $5.2 \pm 0.2 a$ & $5.2 \pm 0.3 a$ & $5.3 \pm 0.2 b$ & $5.2 \pm 0.3 a$ & $4.8 \pm 0.4 a$ & $4.7 \pm 0.3 a$ \\
\hline Trial MNA & $7.3 \pm 0.3 b$ & $6.7 \pm 0.3 c$ & $7.2 \pm 0.2 c$ & $7.2 \pm 0.2 b$ & $7.0 \pm 0.2 b$ & $7.2 \pm 0.3 b$ & $7.3 \pm 0.2 c$ & $7.1 \pm 0.1 c$ & $5.9 \pm 0.2 c$ & $6.2 \pm 0.3 b$ & $6.0 \pm 0.3 b$ & $5.9 \pm 0.5 b$ \\
\hline Trial MNB & $0.8 \pm 0.4 a$ & $1.3 \pm 0.5 a$ & $3.2 \pm 0.2 \mathrm{a}$ & $4.2 \pm 0.3 a$ & $4.5 \pm 0.4 a$ & $5.8 \pm 0.3 a$ & $6.0 \pm 0.3 b$ & $5.8 \pm 0.3 b$ & $4.7 \pm 0.1 a$ & $4.8 \pm 0.4 a$ & $5.8 \pm 0.2 b$ & $5.9 \pm 0.3 b$ \\
\hline \multicolumn{13}{|l|}{ DRBC 2012} \\
\hline Trial MCA & $4.6 \pm 0.3 a$ & $4.1 \pm 0.2 a$ & $4.7 \pm 0.2 \mathrm{a}$ & $6.7 \pm 0.2 c$ & $6.1 \pm 0.2 \mathrm{a}$ & $6.7 \pm 0.1 b$ & $6.4 \pm 0.1 b$ & $5.6 \pm 0.2 a$ & $5.5 \pm 0.2 b$ & $5.8 \pm 0.3 b$ & $4.5 \pm 0.3 a$ & $4.6 \pm 0.3 a$ \\
\hline Trial MCB & $4.3 \pm 0.1 \mathrm{a}$ & $3.9 \pm 0.1 \mathrm{a}$ & $5.8 \pm 0.1 b$ & $5.2 \pm 0.1 \mathrm{a}$ & $5.9 \pm 0.3 a$ & $5.3 \pm 0.3 a$ & $5.6 \pm 0.2 a$ & $5.5 \pm 0.1 \mathrm{a}$ & $5.0 \pm 0.3 a$ & $5.2 \pm 0.2 a$ & $4.8 \pm 0.1 \mathrm{a}$ & $5.9 \pm 0.1 b$ \\
\hline Trial MNA & $4.0 \pm 0.3 a$ & $3.7 \pm 0.3 a$ & $5.0 \pm 0.1 \mathrm{a}$ & $6.0 \pm 0.2 b$ & $6.0 \pm 0.1 a$ & $6.5 \pm 0.0 \mathrm{~b}$ & $5.9 \pm 0.2 a$ & $6.4 \pm 0.2 b$ & $5.4 \pm 0.2 b$ & $6.0 \pm 0.3 b$ & $5.1 \pm 0.2 \mathrm{a}$ & $4.1 \pm 0.2 a$ \\
\hline Trial MNB & $4.0 \pm 0.2 \mathrm{a}$ & $3.5 \pm 0.1 \mathrm{a}$ & $5.6 \pm 0.2 b$ & $5.3 \pm 0.3 a$ & $5.7 \pm 0.2 a$ & $5.0 \pm 0.3 a$ & $5.8 \pm 0.4 a$ & $5.6 \pm 0.3 a$ & $4.8 \pm 0.2 a$ & $5.4 \pm 0.2 a$ & $5.1 \pm 0.2 a$ & $5.7 \pm 0.3 b$ \\
\hline \multicolumn{13}{|l|}{ DRBC 2013} \\
\hline Trial MCA & $4.9 \pm 0.1 b$ & $4.3 \pm 0.3 a$ & $4.1 \pm 0.3 a$ & $4.7 \pm 0.2 b$ & $5.7 \pm 0.2 a$ & $5.7 \pm 0.3 a$ & $4.3 \pm 0.1 a$ & $4.2 \pm 0.3 a$ & $4.9 \pm 0.3 b$ & $5.4 \pm 0.1 b$ & $5.6 \pm 0.3 a$ & $4.2 \pm 0.1 a$ \\
\hline Trial MCB & $4.9 \pm 0.2 b$ & $3.9 \pm 0.1 a$ & $5.8 \pm 0.3 c$ & $5.2 \pm 0.2 b$ & $5.9 \pm 0.3 a$ & $5.3 \pm 0.1 \mathrm{a}$ & $5.0 \pm 0.2 a$ & $5.5 \pm 0.2 c$ & $6.2 \pm 0.2 c$ & $4.3 \pm 0.1 a$ & $5.0 \pm 0.2 a$ & $6.0 \pm 0.1 c$ \\
\hline Trial MNA & $4.2 \pm 0.1 a$ & $4.4 \pm 0.1 a$ & $4.5 \pm 0.2 \mathrm{a}$ & $4.1 \pm 0.2 a$ & $5.8 \pm 0.1 a$ & $5.5 \pm 0.1 \mathrm{a}$ & $4.6 \pm 0.3 a$ & $5.0 \pm 0.3 b$ & $5.0 \pm 0.3 b$ & $5.0 \pm 0.2 b$ & $5.2 \pm 0.4 a$ & $4.1 \pm 0.3 a$ \\
\hline Trial MNB & $4.0 \pm 0.2 \mathrm{a}$ & $4.2 \pm 0.2 a$ & $5.1 \pm 0.1 b$ & $5.0 \pm 0.3 b$ & $5.9 \pm 0.3 a$ & $5.5 \pm 0.2 \mathrm{a}$ & $5.3 \pm 0.2 a$ & $5.6 \pm 0.3 c$ & $4.2 \pm 0.3 a$ & $4.4 \pm 0.3 a$ & $4.8 \pm 0.3 a$ & $5.3 \pm 0.2 b$ \\
\hline \multicolumn{13}{|l|}{ VRBGA 2012} \\
\hline Trial MCA & n.d. & $1.2 \pm 0.0 \mathrm{~b}$ & $0.9 \pm 0.4 a$ & n.d. & n.d. & n.d. & n.d. & n.d. & n.d. & n.d. & n.d. & n.d. \\
\hline Trial MCB & n.d. & $2.0 \pm 0.1 c$ & $2.1 \pm 0.3 b$ & $1.0 \pm 0.1 \mathrm{a}$ & $0.6 \pm 0.2$ & n.d. & n.d. & n.d. & n.d. & n.d. & n.d. & n.d. \\
\hline Trial MNA & n.d. & n.d. & n.d. & n.d. & n.d. & n.d. & n.d. & n.d. & n.d. & n.d. & n.d. & n.d. \\
\hline Trial MNB & n.d. & $0.9 \pm 0.3 a$ & $1.2 \pm 0.2 \mathrm{a}$ & $1.9 \pm 0.1 b$ & n.d. & n.d. & n.d. & n.d. & n.d. & n.d. & n.d. & n.d. \\
\hline \multicolumn{13}{|l|}{ VRBGA 2013} \\
\hline Trial MCA & n.d. & $1.1 \pm 0.1 \mathrm{a}$ & $1.1 \pm 0.3 a$ & n.d. & n.d. & n.d. & n.d. & n.d. & n.d. & n.d. & n.d. & n.d. \\
\hline Trial MCB & n.d. & $2.1 \pm 0.2 b$ & $2.2 \pm 0.4 b$ & $3.0 \pm 0.1 a$ & $1.8 \pm 0.2$ & $0.6 \pm 0.2$ & n.d. & n.d. & n.d. & n.d. & n.d. & n.d. \\
\hline Trial MNA & n.d. & n.d. & n.d. & n.d. & n.d. & n.d. & n.d. & n.d. & n.d. & n.d. & n.d. & n.d. \\
\hline Trial MNB & n.d. & $1.2 \pm 0.2 \mathrm{a}$ & $1.0 \pm 0.3 a$ & $2.8 \pm 0.2 a$ & n.d. & n.d. & n.d. & n.d. & n.d. & n.d. & n.d. & n.d. \\
\hline \multicolumn{13}{|l|}{ РАВ 2012} \\
\hline Trial MCA & n.d. & $2.1 \pm 0.2 a$ & $2.0 \pm 0.1 \mathrm{a}$ & $0.9 \pm 0.1 a$ & $0.3 \pm 0.1 a$ & n.d. & n.d. & n.d. & n.d. & n.d. & n.d. & n.d. \\
\hline Trial MCB & n.d. & $2.4 \pm 0.2 a$ & $3.3 \pm 0.3 b$ & $4.1 \pm 0.3 b$ & $4.4 \pm 0.1 b$ & $5.5 \pm 0.3 a$ & $5.5 \pm 0.2 b$ & $5.1 \pm 0.1 b$ & n.d. & n.d. & n.d. & n.d. \\
\hline Trial MNA & n.d. & $1.8 \pm 0.3 a$ & $1.7 \pm 0.3 a$ & n.d. & n.d. & n.d. & n.d. & n.d. & n.d. & n.d. & n.d. & n.d. \\
\hline Trial MNB & n.d. & $2.1 \pm 0.2 a$ & $3.1 \pm 0.4 b$ & $3.9 \pm 0.4 b$ & $4.2 \pm 0.2 b$ & $5.2 \pm 0.3 a$ & $5.0 \pm 0.3 a$ & $4.6 \pm 0.3 a$ & n.d. & n.d. & n.d. & n.d. \\
\hline \multicolumn{13}{|l|}{ PAB 2013} \\
\hline Trial MCA & n.d. & $2.2 \pm 0.2 b$ & $2.6 \pm 0.1 b$ & $3.9 \pm 0.0 \mathrm{~b}$ & $4.9 \pm 0.2 b$ & $3.3 \pm 0.3 b$ & $3.0 \pm 0.3 a$ & $2.2 \pm 0.4 a$ & n.d. & n.d. & n.d. & n.d. \\
\hline Trial MCB & n.d. & $3.5 \pm 0.2 c$ & $4.3 \pm 0.3 c$ & $5.6 \pm 0.1 d$ & $5.9 \pm 0.1 c$ & $6.0 \pm 0.2 c$ & $6.0 \pm 0.3 b$ & $4.2 \pm 0.3 b$ & n.d. & n.d. & n.d. & n.d. \\
\hline Trial MNA & n.d. & $1.6 \pm 0.2 a$ & $1.1 \pm 0.1 \mathrm{a}$ & $2.0 \pm 0.2 a$ & $4.0 \pm 0.2 a$ & $1.9 \pm 0.2 \mathrm{a}$ & n.d. & n.d. & n.d. & n.d. & n.d. & n.d. \\
\hline Trial MNB & n.d. & $3.7 \pm 0.1 c$ & $2.3 \pm 0.3 b$ & $4.7 \pm 0.1 c$ & $5.5 \pm 0.2 c$ & $5.7 \pm 0.3 c$ & $5.6 \pm 0.3 b$ & $3.8 \pm 0.2 b$ & n.d. & n.d. & n.d. & n.d. \\
\hline $\begin{array}{l}\text { Results of mI } \\
\text { inoculated wi } \\
\text { OM13 and m } \\
\text { rose bengal } \\
\text { pseudomona } \\
\text { and the same } \\
\text { limit of the m }\end{array}$ & $\begin{array}{l}\text { L. pentosus } \\
\text { ually harve } \\
\text { orampheni } \\
\text { n.d., not d } \\
\text { edium }(P< \\
\text { lod. }\end{array}$ & $\begin{array}{l}\text { ed; MNB, } \\
\text { l agar for } \\
\text { cted (valu } \\
\text { 05). Loads }\end{array}$ & $\begin{array}{l}\text { ve drupes } \\
\text { al yeasts } \\
\text { detectior } \\
\text { BP agar }\end{array}$ & $\begin{array}{l}\text { inoculate } \\
\text { filament } \\
\text { nit of mett } \\
\text { e not bee }\end{array}$ & $\begin{array}{l}\text { fungi; } \\
\text { ); differer } \\
\text { ported ir }\end{array}$ & $\begin{array}{l}\text { harveste } \\
\text { A, violet } \\
\text { tters }(a-c) \\
\text { e table sin }\end{array}$ & $\begin{array}{l}\text { MRS, Man } \\
\text { bile gluco } \\
\text { dicate sign } \\
\text { all sample }\end{array}$ & $\begin{array}{l}\text { gosa-Sh } \\
\text { agar for } \\
\text { ant differ } \\
\text { lowed co }\end{array}$ & $\begin{array}{l}\text { agar fo } \\
\text { bacteri } \\
\text { among } \\
\text { tration }\end{array}$ & $\begin{array}{l}\text { sophilic } \\
\text { e; PAB, PS } \\
\text { erimenta } \\
\text { aphyloco }\end{array}$ & $\begin{array}{l}\text { LAB; DR } \\
\text { omonas } \\
\text { Is for the } \\
\text { ess than }\end{array}$ & $\begin{array}{l}\text { drupes } \\
\text { pentosus } \\
\text { lichloran } \\
\text { base for } \\
\text { e sample } \\
\text { letection }\end{array}$ \\
\hline
\end{tabular}


Table 2. Molecular identification of $L A B$ species during the table olive production

\begin{tabular}{|c|c|c|c|c|c|c|c|}
\hline \multirow{2}{*}{$\begin{array}{l}\text { Lactobacillus } \\
\text { species }\end{array}$} & \multirow[b]{2}{*}{ Strain } & \multirow{2}{*}{$\begin{array}{l}\text { Isolation } \\
\text { source (day } \\
\text { of sampling) }\end{array}$} & \multirow{2}{*}{$\begin{array}{l}\text { Size of multiplex } \\
\text { PCR amplicon }\end{array}$} & \multicolumn{2}{|c|}{$\begin{array}{l}\% \text { similarity }{ }^{\mathrm{b}} \\
\text { (accession no. of closest relative) by: }\end{array}$} & \multirow{2}{*}{$\begin{array}{l}\text { Sequence } \\
\text { length (bp) }\end{array}$} & \multirow[b]{2}{*}{ Acc. no. } \\
\hline & & & & BLAST & EzTaxon & & \\
\hline \multirow[t]{5}{*}{ L. pentosus } & 2OCRBL80 & MCA (70d) & 218 & 99 (KF923751.1) & 99.60 (D79211) & 1512 & KP256078 \\
\hline & 2OCRBL81 & MNB (90d) & 218 & 99 (AB362677.1) & 99.60 (D79211) & 1512 & KP256077 \\
\hline & 1OCRBL388 & MNA (130d) & 218 & 99 (KF923751.1) & 99.46 (D79211) & 1505 & KP256089 \\
\hline & 1OCRBL492 & MNA (210d) & 218 & 99 (KF923751.1) & 99.13 (D79211) & 1504 & KP256090 \\
\hline & 10CRBL133 & MCB (9d) & 218 & 98 (KF923751.1) & 98.33 (D79211) & 1512 & KP256081 \\
\hline L. plantarum & BL277-1-OC & MNB (30d) & 318 & 99 (KJ802480.1) & 99.20 (ACGZ01000098) & 1513 & KT268295 \\
\hline
\end{tabular}

were analysed. For these olives, pseudomonads showed lower levels than those estimated for the olives subjected to mechanical harvesting, while Enterobacteriaceae could not be detected. The brines analysed before addition did not host any of these microbial groups. None of the microbial groups previously reported were at detectable levels in the brines added for fermentation.

Owing to the use of the starter culture, trials MCA and MNA showed higher concentrations of $L A B$ than MCB and MNB, whereas an opposite trend was registered for the growth of potential spoilage microorganisms, which was estimated at very low levels for the inoculated trials. Immediately after the inoculation of the starter culture, LAB were about $7.0 \mathrm{log} C F U \mathrm{~mL}^{-1}$ in both trials MCA and MNA. LAB were significantly higher than yeasts. Up to the 130 day, trials MCA and MNA showed a significant increase of both groups. Conversely, trials MCB and MNB showed the lowest $L A B$ and yeast concentrations during the entire period of observations in both years. From day 130 onwards, LAB and yeasts showed almost constant concentration values until the end of the observations. Trial MCB showed the highest concentration of Enterobacteriaceae (around day 9) and pseudomonads (around day 50) in both years. Staphylococci and CPS were not detected in any sample.

Isolation, genotypic characterization and distribution of LAB A total of 1742 colonies were collected from the highest plated dilutions of cell suspensions, and 1481 rods were considered presumptive $L A B$ cultures, as being Gram positive and catalase negative. All cultures were able to grow at $15^{\circ} \mathrm{C}$, unable to develop at $45^{\circ} \mathrm{C}$ and were facultatively homofermentative (grew in the presence of pentose carbohydrates, but $\mathrm{CO}_{2}$ was not produced from glucose). Owing to the high number of isolates, about $40 \%$ of cultures (selected on the basis of the isolation source, colony morphology, experimental trial and year of production) were subjected to RAPD analysis, which allowed the identification of 23 different strains. Results from multiplex PCR analysis and 16S rRNA gene sequencing revealed the presence of a major group of L. pentosus (Table 2) composed of 20 strains (Fig. 1). The other three strains were identified as $L$. plantarum.

Lactobacillus pentosus dominated the LAB population in all trials and in both years of observation. Lactobacillus plantarum was mainly isolated between 50 and 70 days in both spontaneously fermented trials.

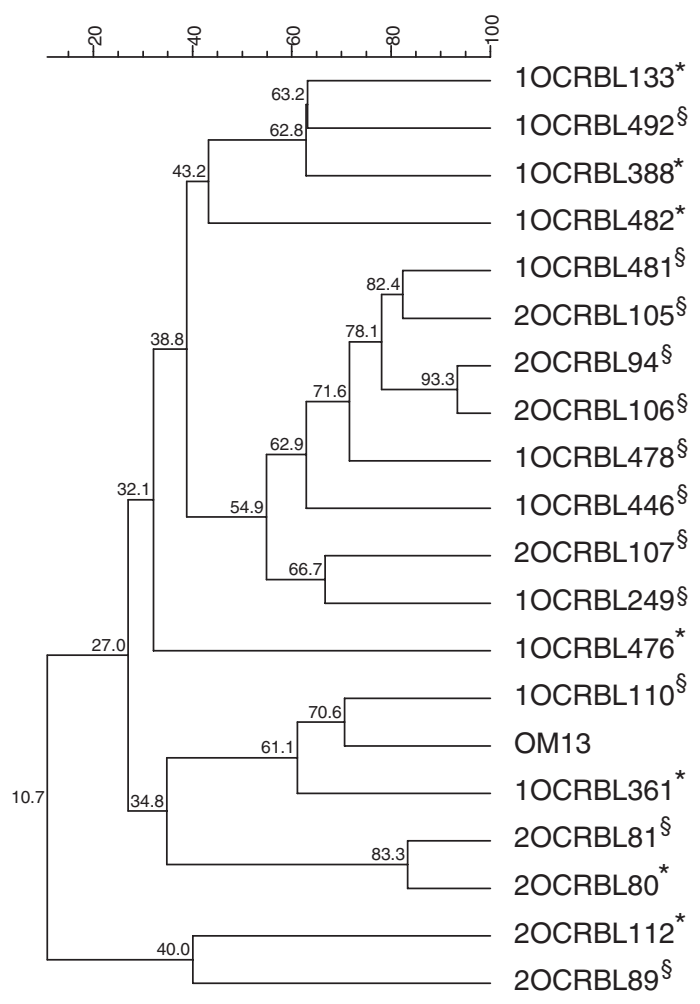

Figure 1. Dendrogram of Lactobacillus pentosus strains isolated during table olive preparation in both years. Symbols: *strains isolated during year 2012; §strains isolated during year 2013; OM13, commercial starter culture.

In terms of biodiversity within the L. pentosus species, trials MCB and MNB showed the highest number of strains. As expected, the commercial starter OM13 inoculated in MCA and in MNA was the strain most frequently isolated during fermentation in both trials and in both years. No L. pentosus strain of year 2012 was then found during year 2013.

\section{Isolation, identification and distribution of yeasts}

A total of 3108 yeast colonies were collected from DRBC agar. Based on colony and cell morphology, 593 isolates were subjected to molecular identification. After restriction analysis of 5.8S-ITS region, the isolates were clustered into 11 groups (Table 3 ). 
Table 3. Molecular identification of yeasts isolated during table olive production

\begin{tabular}{|c|c|c|c|c|c|c|c|c|c|}
\hline \multirow[b]{2}{*}{ Species } & \multirow[b]{2}{*}{ Strain } & \multirow{2}{*}{$\begin{array}{l}\text { Isolation } \\
\text { source } \\
\text { (day of } \\
\text { sampling) }\end{array}$} & \multirow{2}{*}{\multicolumn{2}{|c|}{ 5.8S-ITS }} & \multicolumn{3}{|c|}{ Size of restriction fragments } & \multirow{2}{*}{$\begin{array}{l}\% \text { similarity }^{a} \\
\text { (accession } \\
\text { no. of closest } \\
\text { relative) by: }\end{array}$} & \multirow[b]{2}{*}{ Acc. no. } \\
\hline & & & & & Cfol & HaellI & Hinfl & & \\
\hline \multirow[t]{5}{*}{ Candida boidinii } & 10CRY307 & MCB (30d) & I & 700 & $329+299$ & 700 & 390 & 99 (KC442246.1) & KP256103 \\
\hline & $10 C R Y 366$ & MCA (90d) & $\|$ & 740 & $346+312+82$ & 733 & $378+182+147$ & 99 ( KC442246.1) & KP256104 \\
\hline & $10 C R Y 264$ & MNA (30d) & III & 780 & $335+302$ & 740 & 377 & $99($ KC442246.1) & KP256105 \\
\hline & $10 C R Y 446$ & MCB (170d) & IV & 750 & $343+309+81$ & 750 & $393+195+158$ & 99 (EU293427.1) & KP256106 \\
\hline & 10CRY300 & MCB (30d) & V & 740 & $329+299$ & 740 & 390 & 99 (GU373760.1) & KP256107 \\
\hline Candida diddensiae & 2OCRY20 & MCA (6d) & $\mathrm{VI}$ & 695 & $\begin{array}{c}300+184+ \\
142+68\end{array}$ & $\begin{array}{c}450+143 \\
\quad+91\end{array}$ & 341 & 99 (U45750.1) & KP256101 \\
\hline $\begin{array}{l}\text { Candida } \\
\quad \text { membranifaciens }\end{array}$ & 1OCRY126 & MNB (6d) & VII & 650 & $314+297$ & $\begin{array}{c}420+150+ \\
80\end{array}$ & 334 & 99 (EF362752.1) & KP256097 \\
\hline \multirow{4}{*}{$\begin{array}{l}\text { Wickerhamomyces } \\
\text { anomalus }\end{array}$} & 10CRY107 & MCB (3d) & VIII & 600 & 600 & 600 & 305 & 99 (KM246030.1) & KP256095 \\
\hline & 10CRY127 & MCA (170d) & IX & 620 & 598 & 619 & 329 & 100 (HM107788.1) & KP256098 \\
\hline & 1OCRY180 & MCA (30d) & $x$ & 650 & 598 & 600 & 331 & 99 (KC510047.1) & KP256099 \\
\hline & 10CRY267 & MNB (50d) & $X I$ & 670 & 583 & 625 & 325 & 99 ( JX049437.1) & KP256100 \\
\hline
\end{tabular}

Sequencing of the D1/D2 domain of the 26S rRNA gene identified seven species: Candida boidinii (groups I-V), Candida diddensiae (group VI), Candida membranifaciens (group VII) and Wickerhamomyces anomalus (groups $\mathrm{VIII-XI).} \mathrm{C} \mathrm{boidinii} \mathrm{and} \mathrm{W.}$ anomalus mainly dominated the yeast population in both years. In detail, $C$. boindii was frequently isolated during the fermentations of MCA and MNA. Blastomycetes isolated from MCB and MNB were mainly represented by $W$. anomalus and $C$. diddensiae; C. membranifaciens was isolated only at day 6 in trial MNB (year 2012).

\section{Analysis of VOCs and sensory evaluation}

The results of VOC analyses carried out on olive samples at day 210 are reported in Table 4 for both years. Acids, alcohols and aldehydes were detected at the highest concentrations in both campaigns. Hydrocarbons reached high concentrations in 2012. Among acids, acetic, hexadecanoic, nonanoic, butanoic and octanoic acids showed the highest values in MNA and MNB; the main compounds within the class of alcohols were phenylethyl alcohol, 1-butanol-3-methyl and benzyl alcohol. cis-Hexen-1-ol was estimated at high concentration in MCA and MCB in 2013. Aldehydes were mainly represented by 2-decenal (E), octanal and benzaldehyde; on the other hand, hydrocarbons and phenols were represented by squalene and homoguaiocol, respectively.

Results of the sensory analysis are reported in Table 5. Significant $(P<0.05)$ differences were mainly found among the trials. The main differences were estimated in terms of green olive aroma, complexity (odour), bitterness, complexity and off-flavours (taste). In detail, the MCA and MNA trials showed the highest scores for green colour intensity, green olive aroma and taste complexity, and the lowest scores for bitter and astringent taste. On the other hand, MCB showed values of off-odours and off-flavours significantly higher than the other trials.

\section{Statistical and explorative multivariate analysis}

The combined ANOVA over years 2012 and 2013 showed no significant $(P<0.05)$ interactions between years and the dependent variables corresponding to $\mathrm{pH}$ values and microbial loads detected on MRS, DRBC and BP media.

HCA classified the trials in accordance with their mutual dissimilarity and relationship using the 18 variables selected on the basis of results from $\mathrm{pH}$ determination and microbial analysis (Fig. 2). In both years, replicates of all trials were clearly separated into two mega-clusters (MCA, MNA and MCB, MNB) in accordance with method of fermentation and regardless of harvesting technique. Furthermore, within each mega-cluster, two groups at a low level of dissimilarity were found on the basis of the fermentation method. The lowest level of dissimilarity was estimated among trials MNA and MCA in 2012.

The results obtained by monitoring microbial counts and $\mathrm{pH}$ values were also subjected to PCA (Fig. 3). The components of the PCA were correlated with variables as shown in Fig. 3(a) (2012) and Fig. 3(c) (2013), and the corresponding values of factor loadings are reported in Table S1 (supporting information). The discrimination of trials can be visualized in the plot of the scores (Fig. 3b, d). In detail, Fig. 3(b) shows the projection of the cases (representing the three replicates per each trials) on to the planes as a function of factors 1 and 2 (66.96\% of the total variance explained), in 2012. The trials were significantly separated along factor 1 on the basis of the fermentation method, regardless of the harvest technique. These results confirmed those obtained by HCA. Furthermore, in 2013, the trials MCA and MNA were closely related to one another also along factor 2 (Fig. 3d).

With regard to VOCs, PCA results are shown in Fig. 4. In order to construct a graphical representation of loading (Fig. 4a, c) and score (Fig. 4b, d) plots, the first two components F1 and F2 were selected. The score plots (Fig. 4b, d) clearly represented the four trials in separated areas of PCA quadrants. The harvest techniques mainly affected the composition of VOCs for the samples in 2013, 
Table 4. Concentration of volatile organic compounds $\left(\mu \mathrm{g} \mathrm{kg}^{-1}\right.$ ) at the end (day 210) of olive production during both 2012 and 2013 campaigns

\begin{tabular}{|c|c|c|c|c|c|c|c|c|}
\hline \multirow[b]{2}{*}{ Compound } & \multicolumn{4}{|c|}{2012 campaign } & \multicolumn{4}{|c|}{2013 campaign } \\
\hline & Trial MCA & Trial MCB & Trial MNA & Trial MNB & Trial MCA & Trial MCB & Trial MNA & Trial MNB \\
\hline Acetic acid & $5448.1 \pm 206.0$ & $6145.0 \pm 218.9$ & $9530.5 \pm 308.9$ & $6747.9 \pm 213.4$ & $6374.2 \pm 192.1$ & $7742.8 \pm 305.8$ & $10907.8 \pm 218.3$ & $5305.0 \pm 177.3$ \\
\hline Butanoic acid & $219.8 \pm 21.9$ & $239.2 \pm 28.0$ & $300.2 \pm 22.3$ & $257.1 \pm 15.2$ & $258.8 \pm 24.0$ & $301.4 \pm 35.3$ & $137.9 \pm 19.2$ & $20.2 \pm 2.6$ \\
\hline Heptanoic acid & n.d. & n.d. & n.d. & n.d. & $22.6 \pm 1.1$ & $19.3 \pm 2.9$ & $45.3 \pm 6.3$ & $17.3 \pm 1.0$ \\
\hline Hexadecanoic acid & $758.5 \pm 75.4$ & $1312.6 \pm 153.6$ & $1797.4 \pm 133.7$ & $1896.6 \pm 112.2$ & $1031.1 \pm 110.3$ & $1653.9 \pm 193.5$ & $498.1 \pm 69.3$ & $176.5 \pm 22.5$ \\
\hline Hexanoic acid & $577.2 \pm 129.1$ & $249.6 \pm 29.2$ & $268.3 \pm 25.5$ & $212.5 \pm 13.0$ & $121.5 \pm 26.4$ & $100.4 \pm 11.7$ & $57.6 \pm 8.0$ & $79.7 \pm 3.2$ \\
\hline 2-Ethylhexanoic acid & n.d. & n.d. & n.d. & n.d. & $7.9 \pm 0.4$ & $10.5 \pm 2.3$ & $26.6 \pm 3.7$ & $13.2 \pm 0.4$ \\
\hline Nonanoic acid & $1170.1 \pm 230.1$ & $651.3 \pm 76.2$ & $483.1 \pm 46.0$ & $275.0 \pm 48.5$ & $160.6 \pm 30.5$ & $81.7 \pm 9.6$ & $256.3 \pm 35.7$ & $94.5 \pm 12.1$ \\
\hline Octanoic acid & $1051.5 \pm 206.8$ & $437.8 \pm 51.2$ & $408.3 \pm 38.9$ & $273.1 \pm 21.9$ & $162.9 \pm 29.2$ & $54.3 \pm 6.4$ & $57.6 \pm 8.0$ & $107.5 \pm 13.7$ \\
\hline Pentanoic acid & $475.9 \pm 90.5$ & $306.7 \pm 35.9$ & $308.3 \pm 29.3$ & $197.0 \pm 15.8$ & $73.8 \pm 13.2$ & $38.7 \pm 4.5$ & $59.7 \pm 8.3$ & $11.6 \pm 1.5$ \\
\hline Propionic acid & $213.6 \pm 11.1$ & $76.3 \pm 8.9$ & $180.6 \pm 23.5$ & $51.1 \pm 4.1$ & $242.8 \pm 50.6$ & $96.2 \pm 11.2$ & $151.6 \pm 21.1$ & $94.8 \pm 12.1$ \\
\hline 1,4-Butanediol & $38.6 \pm 7.2$ & $72.1 \pm 10.0$ & $55.6 \pm 5.9$ & $53.5 \pm 10.3$ & $61.6 \pm 4.8$ & $90.9 \pm 12.5$ & n.d. & $12.0 \pm 1.0$ \\
\hline 1-Butanol-3-methyl & $295.7 \pm 49.8$ & $477.4 \pm 65.9$ & $686.2 \pm 72.3$ & $1506.2 \pm 458.7$ & $399.0 \pm 47.5$ & $601.5 \pm 83.1$ & $358.3 \pm 25.0$ & $286.3 \pm 23.9$ \\
\hline 1-Hexanol & $108.3 \pm 18.2$ & $132.2 \pm 16.0$ & $131.3 \pm 13.8$ & $132.8 \pm 23.4$ & $172.0 \pm 47.6$ & $166.6 \pm 20.2$ & $145.7 \pm 10.2$ & $203.6 \pm 13.4$ \\
\hline 1-Octanol & $851.8 \pm 143.4$ & $201.6 \pm 27.8$ & $289.9 \pm 16.2$ & $168.0 \pm 26.9$ & $1149.3 \pm 136.9$ & $254.0 \pm 35.1$ & $776.7 \pm 54.2$ & $143.0 \pm 7.5$ \\
\hline 2-Nonen-1-ol & $762.1 \pm 31.5$ & $374.3 \pm 51.7$ & $423.5 \pm 23.7$ & $350.8 \pm 56.2$ & $195.3 \pm 14.0$ & $471.7 \pm 65.2$ & $25.7 \pm 1.8$ & $40.9 \pm 1.7$ \\
\hline Benzyl alcohol & $602.2 \pm 24.9$ & $493.4 \pm 68.2$ & $672.6 \pm 32.3$ & $150.7 \pm 24.1$ & $643.3 \pm 46.3$ & $621.7 \pm 85.9$ & $707.9 \pm 49.4$ & $648.4 \pm 38.4$ \\
\hline cis-Hexen-1-ol & $728.2 \pm 14.5$ & $575.6 \pm 79.5$ & $326.8 \pm 15.7$ & $32.9 \pm 2.2$ & $759.8 \pm 54.7$ & $725.2 \pm 21.2$ & $493.8 \pm 38.7$ & $345.2 \pm 20.4$ \\
\hline Phenylethyl alcohol & $1001.4 \pm 252.0$ & $1374.6 \pm 189.9$ & $1998.7 \pm 96.1$ & $1824.0 \pm 321.6$ & $1783.5 \pm 432.3$ & $1732.0 \pm 239.3$ & $1411.6 \pm 164.46$ & $945.3 \pm 57.0$ \\
\hline 2-Butenal-2-methyl & $39.1 \pm 1.5$ & $45.0 \pm 2.0$ & $49.7 \pm 2.4$ & $58.1 \pm 1.3$ & $38.2 \pm 1.7$ & $42.3 \pm 1.8$ & $29.8 \pm 1.6$ & $17.1 \pm 1.1$ \\
\hline 2-Decenal (E) & $1672.8 \pm 66.1$ & $351.3 \pm 15.3$ & $832.8 \pm 115.9$ & $387.2 \pm 21.8$ & $1634.7 \pm 24.4$ & $330.2 \pm 14.4$ & $1620.9 \pm 148.4$ & $125.3 \pm 8.2$ \\
\hline Benzaldehyde & $85.4 \pm 3.4$ & $579.5 \pm 25.3$ & $345.7 \pm 19.3$ & $304.6 \pm 51.4$ & $83.4 \pm 10.4$ & $544.7 \pm 23.8$ & $116.5 \pm 19.3$ & $372.0 \pm 59.6$ \\
\hline Benzaldehyde-2,5-dimethyl & $85.4 \pm 3.4$ & $579.5 \pm 25.3$ & $345.7 \pm 19.3$ & $304.6 \pm 61.3$ & $19.9 \pm 2.3$ & $54.6 \pm 2.4$ & $14.5 \pm 2.4$ & $34.7 \pm 1.0$ \\
\hline Benzaldehyde-3-ethyl & n.d. & $43.1 \pm 1.9$ & $21.8 \pm 1.4$ & $46.2 \pm 2.6$ & $20.7 \pm 2.7$ & $40.6 \pm 1.8$ & $20.8 \pm 3.4$ & $23.2 \pm 0.5$ \\
\hline Nonanal & $201.1 \pm 7.9$ & $207.0 \pm 9.0$ & $272.2 \pm 18.1$ & $389.0 \pm 21.9$ & $196.6 \pm 2.7$ & $194.6 \pm 4.8$ & $181.1 \pm 13.0$ & $196.3 \pm 3.8$ \\
\hline Octanal & $701.8 \pm 12.3$ & $169.1 \pm 7.4$ & $229.3 \pm 33.9$ & $100.7 \pm 2.3$ & $708.9 \pm 14.1$ & $158.9 \pm 6.9$ & $285.4 \pm 5.9$ & $1044.3 \pm 20.5$ \\
\hline Phenylacetaldehyde & $29.6 \pm 0.5$ & $71.5 \pm 3.1$ & $72.4 \pm 4.0$ & $89.1 \pm 2.0$ & $41.1 \pm 1.1$ & $19.2 \pm 1.0$ & $39.7 \pm 1.9$ & $60.9 \pm 1.2$ \\
\hline Vanillin & $146.8 \pm 21.0$ & $115.4 \pm 5.0$ & n.d. & n.d. & $208.6 \pm 5.3$ & $108.4 \pm 4.7$ & $136.6 \pm 2.3$ & $76.3 \pm 1.5$ \\
\hline 2-Nonanone & $267.1 \pm 4.7$ & $167.9 \pm 5.9$ & $105.7 \pm 5.5$ & $76.5 \pm 1.5$ & $269.8 \pm 7.4$ & $157.8 \pm 5.6$ & $46.3 \pm 0.8$ & n.d. \\
\hline 3-Hydroxybutanone & n.d. & n.d. & $151.4 \pm 7.8$ & $123.6 \pm 2.4$ & $18.5 \pm 2.1$ & $21.8 \pm 0.8$ & $33.8 \pm 0.6$ & $29.7 \pm 1.6$ \\
\hline 4-Ethylacetophenone & n.d. & n.d. & n.d. & n.d. & $7.9 \pm 0.9$ & n.d. & $11.2 \pm 0.9$ & $54.9 \pm 2.9$ \\
\hline 4-Methyldihydro-2-(3H)-furanone & n.d. & n.d. & n.d. & n.d. & n.d. & $5.7 \pm 0.8$ & $107.7 \pm 8.4$ & $84.8 \pm 4.6$ \\
\hline Butyrolactone & $345.6 \pm 20.6$ & $415.7 \pm 57.4$ & n.d. & n.d. & $97.3 \pm 3.0$ & $90.4 \pm 2.5$ & $43.1 \pm 3.4$ & $51.7 \pm 2.8$ \\
\hline Cyclopentanone & n.d. & n.d. & $345.1 \pm 17.9$ & $233.4 \pm 4.6$ & $75.6 \pm 2.1$ & $22.1 \pm 1.1$ & $99.5 \pm 1.7$ & $94.9 \pm 5.0$ \\
\hline Benzyl acetate & n.d. & n.d. & n.d. & n.d. & n.d. & n.d. & $64.5 \pm 7.4$ & $68.2 \pm 1.6$ \\
\hline cis-3-Hexenylacetate & n.d. & n.d. & n.d. & n.d. & n.d. & n.d. & $35.3 \pm 1.8$ & $35.0 \pm 1.9$ \\
\hline Ethyl dihydrocinnamate & n.d. & n.d. & n.d. & n.d. & n.d. & n.d. & $30.9 \pm 0.9$ & n.d. \\
\hline Ethyl lactate & n.d. & n.d. & n.d. & n.d. & n.d. & n.d. & $37.7 \pm 1.7$ & $16.3 \pm 2.7$ \\
\hline Methyl hexadecanoate & n.d. & n.d. & n.d. & n.d. & n.d. & n.d. & $266.7 \pm 47.1$ & $133.2 \pm 22.4$ \\
\hline Methyl hydrocinnamate & n.d. & n.d. & n.d. & n.d. & n.d. & n.d. & $15.5 \pm 2.7$ & $91.3 \pm 15.4$ \\
\hline Methyl salicylate & n.d. & n.d. & n.d. & n.d. & n.d. & n.d. & $44.9 \pm 7.9$ & $31.5 \pm 1.3$ \\
\hline Octyl acetate & n.d. & n.d. & n.d. & n.d. & n.d. & n.d. & $95.4 \pm 16.8$ & $87.0 \pm 3.9$ \\
\hline 4-Ethylphenol & n.d. & n.d. & n.d. & n.d. & $87.7 \pm 1.7$ & n.d. & $212.9 \pm 16.6$ & $224.5 \pm 35.8$ \\
\hline Guaiacol & $242.2 \pm 2.7$ & $3901.8 \pm 539.0$ & $458.4 \pm 23.7$ & $7161.8 \pm 296.4$ & $242.2 \pm 2.4$ & $491.5 \pm 7.9$ & $141.9 \pm 11.1$ & $149.8 \pm 5.1$ \\
\hline Homoguaiacol & $1850.7 \pm 219.0$ & $874.8 \pm 120.8$ & $2601.0 \pm 145.3$ & n.d. & $2257.9 \pm 267.2$ & $1102.3 \pm 152.3$ & $1775.3 \pm 138.6$ & $1773.8 \pm 223.2$ \\
\hline Phenol & $642.2 \pm 37.1$ & $758.8 \pm 14.8$ & $374.5 \pm 18.0$ & $811.7 \pm 33.6$ & $115.3 \pm 10.7$ & $956.2 \pm 12.1$ & $51.5 \pm 4.0$ & $57.3 \pm 3.1$ \\
\hline$\alpha$-Terpineol & n.d. & n.d. & n.d. & n.d. & n.d. & n.d. & $28.9 \pm 5.1$ & $26.2 \pm 1.3$ \\
\hline Squalene & $1185.0 \pm 110.1$ & $11820.4 \pm 1032.9$ & $3382.5 \pm 162.7$ & $9779.8 \pm 404.7$ & $1386.4 \pm 128.8$ & $2018.6 \pm 178.8$ & n.d. & n.d. \\
\hline Styrene & n.d. & $94.2 \pm 13.0$ & $114.0 \pm 5.5$ & $181.5 \pm 7.5$ & n.d. & $118.7 \pm 16.4$ & n.d. & n.d. \\
\hline$\alpha$-Cubebene & $107.6 \pm 6.0$ & $148.0 \pm 8.0$ & $132.9 \pm 6.4$ & $56.3 \pm 4.8$ & $122.2 \pm 6.6$ & $162.7 \pm 8.8$ & $39.5 \pm 4.5$ & $78.8 \pm 2.5$ \\
\hline
\end{tabular}




\begin{tabular}{|c|c|c|c|c|c|c|c|c|}
\hline \multirow[b]{2}{*}{ Descriptors } & \multicolumn{4}{|c|}{2012 campaign } & \multicolumn{4}{|c|}{2013 campaign } \\
\hline & Trial MCA & Trial MCB & Trial MNA & Trial MNB & Trial MCA & Trial MCB & Trial MNA & Trial MNB \\
\hline \multicolumn{9}{|l|}{ Aspect } \\
\hline Green colour intensity & $6.2 \pm 0.2 b$ & $5.3 \pm 0.4 a$ & $6.4 \pm 0.1 b$ & $5.9 \pm 0.6 b$ & $6.3 \pm 0.2 b$ & $5.2 \pm 0.2 \mathrm{a}$ & $6.6 \pm 0.3 b$ & $5.8 \pm 0.3 a$ \\
\hline Brightness & $5.7 \pm 0.2 \mathrm{a}$ & $5.5 \pm 0.4 a$ & $5.8 \pm 0.2 a$ & $5.5 \pm 0.1 \mathrm{a}$ & $5.6 \pm 0.4 a$ & $5.1 \pm 0.1 \mathrm{a}$ & $5.8 \pm 0.2 a$ & $5.5 \pm 0.3 a$ \\
\hline \multicolumn{9}{|l|}{ Odour } \\
\hline Green olive aroma & $7.3 \pm 0.3 c$ & $5.5 \pm 0.3 a$ & $7.4 \pm 0.4 c$ & $6.4 \pm 0.3 b$ & $7.5 \pm 0.4 b$ & $5.5 \pm 0.4 a$ & $7.4 \pm 0.4 b$ & $6.6 \pm 0.2 b$ \\
\hline Complexity & $6.3 \pm 0.4 c$ & $4.4 \pm 0.3 a$ & $6.5 \pm 0.3 c$ & $5.5 \pm 0.4 b$ & $5.7 \pm 1.7 b$ & $4.7 \pm 0.3 a$ & $7.0 \pm 0.2 c$ & $5.3 \pm 0.3 b$ \\
\hline Off-odours & $1.1 \pm 0.2 \mathrm{a}$ & $3.4 \pm 0.4 c$ & $1.1 \pm 0.1 \mathrm{a}$ & $2.2 \pm 0.1 b$ & $1.1 \pm 0.2 \mathrm{a}$ & $3.3 \pm 0.3 c$ & $1.0 \pm 0.2 a$ & $2.2 \pm 0.2 b$ \\
\hline \multicolumn{9}{|l|}{ Taste } \\
\hline Crispness & $5.2 \pm 0.1 \mathrm{a}$ & $5.2 \pm 0.1 \mathrm{a}$ & $4.8 \pm 0.1 \mathrm{a}$ & $4.9 \pm 0.1 \mathrm{a}$ & $5.2 \pm 0.1 \mathrm{a}$ & $5.2 \pm 0.2 \mathrm{a}$ & $4.9 \pm 0.2 a$ & $4.8 \pm 0.1 \mathrm{a}$ \\
\hline Easy stone & $3.8 \pm 0.1 \mathrm{a}$ & $4.7 \pm 0.1 \mathrm{a}$ & $3.9 \pm 0.1 \mathrm{a}$ & $4.3 \pm 0.3 a$ & $3.9 \pm 0.1 b$ & $2.4 \pm 0.2 \mathrm{a}$ & $4.2 \pm 0.1 b$ & $3.5 \pm 0.1 b$ \\
\hline Juicy & $5.5 \pm 0.4 b$ & $3.5 \pm 0.4 a$ & $5.3 \pm 0.1 b$ & $4.6 \pm 0.4 a$ & $5.5 \pm 0.4 c$ & $3.5 \pm 0.3 a$ & $5.4 \pm 0.2 c$ & $4.6 \pm 0.4 b$ \\
\hline Sweet & $2.6 \pm 0.4 a$ & $2.1 \pm 0.3 a$ & $2.9 \pm 0.2 \mathrm{a}$ & $2.5 \pm 0.2 \mathrm{a}$ & $2.7 \pm 0.4 b$ & $2.1 \pm 0.3 a$ & $2.9 \pm 0.2 b$ & $2.5 \pm 0.2 b$ \\
\hline Sour & $3.6 \pm 0.4 a$ & $4.4 \pm 0.3 b$ & $3.8 \pm 0.2 \mathrm{a}$ & $4.3 \pm 0.4 b$ & $3.5 \pm 0.3 a$ & $4.2 \pm 0.1 b$ & $4.0 \pm 0.1 a$ & $4.3 \pm 0.4 b$ \\
\hline Bitter & $2.8 \pm 0.4 b$ & $5.0 \pm 0.2 c$ & $2.2 \pm 0.1 a$ & $2.9 \pm 0.2 b$ & $3.4 \pm 0.2 a$ & $5.4 \pm 0.3 b$ & $3.2 \pm 0.1 \mathrm{a}$ & $4.2 \pm 0.0 b$ \\
\hline Salt & $3.4 \pm 0.3 b$ & $3.9 \pm 0.2 b$ & $2.5 \pm 0.2 \mathrm{a}$ & $3.7 \pm 0.0 b$ & $3.2 \pm 0.1 \mathrm{a}$ & $4.2 \pm 0.1 b$ & $3.4 \pm 0.1 \mathrm{a}$ & $4.3 \pm 0.0 \mathrm{~b}$ \\
\hline Complexity & $5.8 \pm 0.3 b$ & $4.0 \pm 0.2 \mathrm{a}$ & $6.6 \pm 0.2 c$ & $5.7 \pm 0.2 b$ & $5.0 \pm 0.0 c$ & $3.2 \pm 0.1 \mathrm{a}$ & $5.2 \pm 0.0 c$ & $4.4 \pm 0.1 b$ \\
\hline Astringent & $2.2 \pm 0.2 \mathrm{a}$ & $3.8 \pm 0.1 b$ & $2.4 \pm 0.1 \mathrm{a}$ & $3.4 \pm 0.1 b$ & $3.1 \pm 0.0 \mathrm{a}$ & $4.1 \pm 0.0 b$ & $3.1 \pm 0.0 \mathrm{a}$ & $3.6 \pm 0.2 a$ \\
\hline Off-flavours & $1.2 \pm 0.1 \mathrm{a}$ & $3.9 \pm 0.1 c$ & $1.3 \pm 0.0 a$ & $3.3 \pm 0.2 b$ & $1.1 \pm 0.1 b$ & $2.7 \pm 0.2 d$ & $0.9 \pm 0.1 \mathrm{a}$ & $2.1 \pm 0.1 c$ \\
\hline
\end{tabular}

Abbreviations: MCA, olive drupes inoculated with L. pentosus OM13 and mechanically harvested; MCB, olive drupes uninoculated and mechanically harvested; MNA, olive drupes inoculated with L. pentosus OM13 and manually harvested; MNB, olive drupes uninoculated and manually harvested. Different letters $(a-d)$ indicate significant differences between experimental trials for the same sample for $P \leq 0.05$.

when trials were significantly separated along the F1 component. The correlation of VOC variables with the main factors of PCA are reported in Tables S2 and S3 for 2012 and 2013, respectively.

The multivariate statistical analysis was concluded by using data of sensory analysis of olives. Biplot graphical representations were constructed as illustrated in Fig. 5(a, b). The four trials resulting mainly separated along the F1 component. Trials MCB and MNB were closely associated with bitter, astringent, salt and off-odour descriptors, in both years. Conversely, MCA and MNA showed the closest correlation with green olive aroma and complexity (odour and taste).

Barlett's sphericity test was applied to all data matrix inputs, and statistically significant differences $(P<0.0001)$ were found among trials.

\section{DISCUSSION}

The aim of the present study was to evaluate the effect of different harvesting methodologies and processing conditions on the quality of fermented table olives of the cultivar Nocellara del Belice. To our knowledge, no study has so far been carried out to evaluate the effects of the mechanical harvesting of drupes on the final characteristics of table olives. Limited information has been published on the rheological characteristics of drupes just after mechanical harvesting, ${ }^{48-50}$ but no data are available on the microbial and chemical features of the product during fermentation.

The results showed that the mechanical harvesting of drupes subjected to the driven fermentation by L. pentosus OM13 as starter culture did not affect negatively the microbial characteristics of the transformation process. However, besides LAB and yeasts, Enterobacteriaceae were also found. These microorganisms, at high concentrations, might spoil the organoleptic quality and safety of table olives acting as alterative and/or pathogenic microorganisms. ${ }^{51,52}$ Many species belonging to the pseudomonad group are commonly identified as food spoilage bacteria, but also as human pathogens. Pseudomonas spp. can be characterized by high levels of proteolitic, lipolitic and pectinolitic activities ${ }^{53,54}$ damaging the final product.

The inoculation of LAB starter into the brines of MCA and MNA significantly reduced the growth of potential spoilage and/or pathogenic microorganisms. The use of selected LAB strains as starters determines a rapid acidification of the product and inhibits the growth of potential spoilage populations. ${ }^{55}$ In this work, both trials inoculated with the starter culture showed a significant decrease of $\mathrm{pH}$ within the sixth day of fermentation, a trend also registered by other authors. ${ }^{56}$ From day 70 onward, and in particular for year 2012, pH values significantly increased. Although LAB clearly dominated the microbial population of the inoculated trials and the production of lactic acid reduced the $\mathrm{pH}$, yeast concentration was estimated at detectable levels until the end of the process. Yeasts might oxidize lactic acid and the increase of $\mathrm{pH}$ might be significant. ${ }^{57}$ Interestingly, the combined analysis of variance of data showed no significant interactions between years and dependent variables, especially for $\mathrm{pH}$ values and $\mathrm{LAB}$ concentrations. These two parameters are defining for the chemical and sensory quality of the final product because they might inhibit significantly the growth of spoilage/pathogenic microorganisms.

Several LAB species might have different influence on the quality of table olives, but the species commonly associated with this production process are $L$. pentosus and $L$. plantarum. ${ }^{16,22,58}$ In our study, $L$. pentosus was the main LAB species found in all trials during both years. This species is commonly recognized as one of the most technologically relevant $L A B$ owing to its high aptitude to ferment olives. For this reason, its dominance during table olive production might represent a guarantee of quality of the final in terms of microbiological and chemical characteristics. 

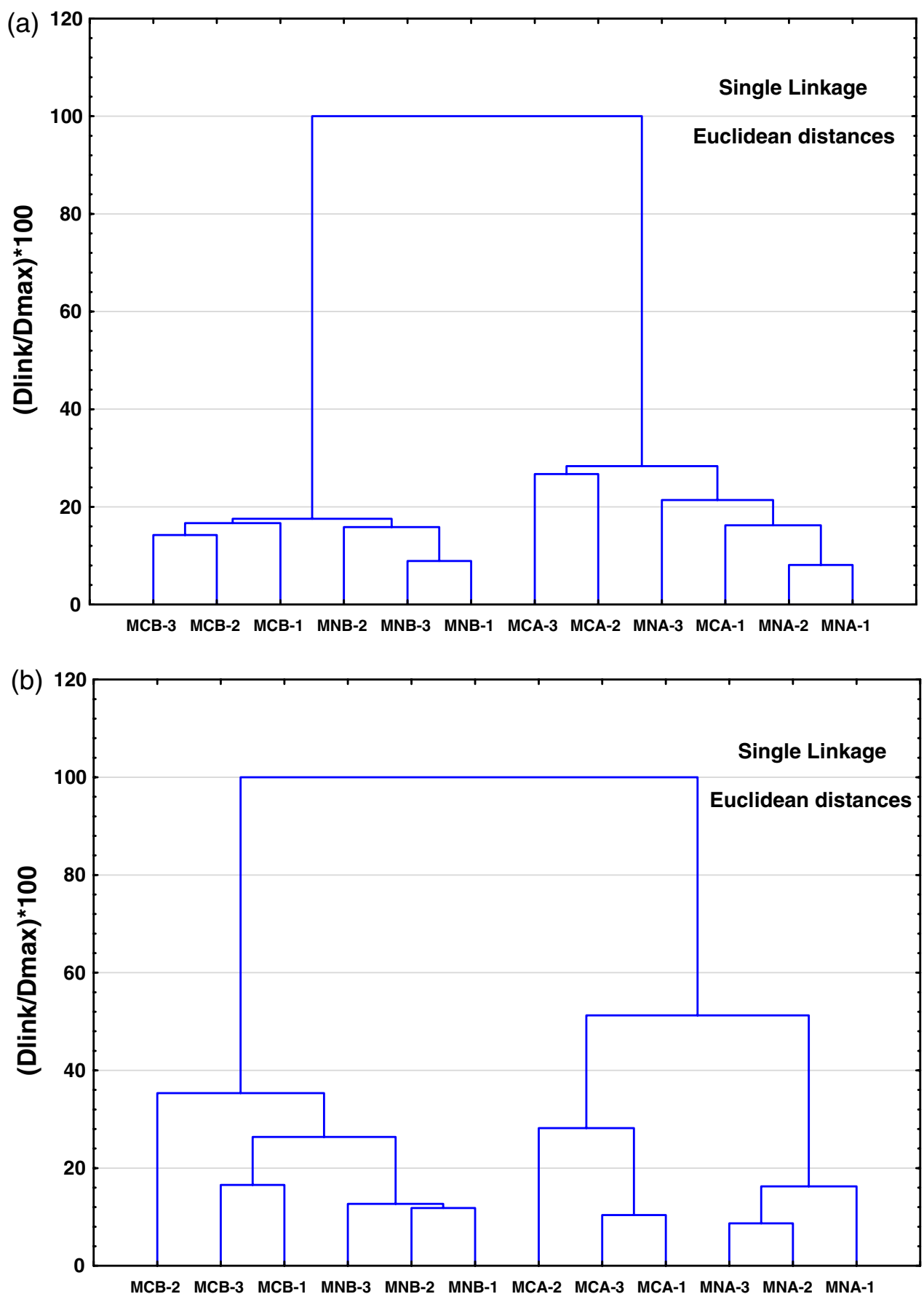

Figure 2. Dendrogram of brine samples resulting from HCA analysis based on values of microbial and pH changes during the 2012 (a) and 2013 (b) campaigns. The dissimilarity among samples was measured by Euclidean distance, whereas cluster aggregation was achieved by single linkage. Abbreviations: MCA, MCB, MNA and MNB correspond to codes used for the experimental trials. The numbers associated with codes correspond to replicates per each experimental trials.

Several autochthonous strains belonging to $L$. pentosus were found during fermentation even in presence of the commercial starter in both years. The presence of several $L$. pentosus strains during olive transformation is reported to improve the complexity of the sensory profile of the final product. . $^{22,42}$

The yeast species isolated in this work are commonly associated with the table olive environment, mostly with the fermentation of green table olives. ${ }^{59,60}$ In particular, the species $W$. anomalus mainly dominated the yeast population during the entire production of MNA and MCB olives. Some previous studies ${ }^{25,61}$ reported that table olive fermentation might be carried out by yeasts. Yeasts are relevant in directly brined green and black natural olive fermentations, where fruits are not treated with sodium hydroxide and LAB are partially inhibited due to the presence of phenolic compounds. ${ }^{30}$ However, an overgrowth of fermentative yeasts could induce a high production of $\mathrm{CO}_{2}$ that could damage drupe texture. ${ }^{62}$

Thus LAB and yeasts in table olives have an essential role in olive preservation. It has been clearly shown in other works on table olives ${ }^{55}$ and/or in other food production ${ }^{63,64}$ that, although 

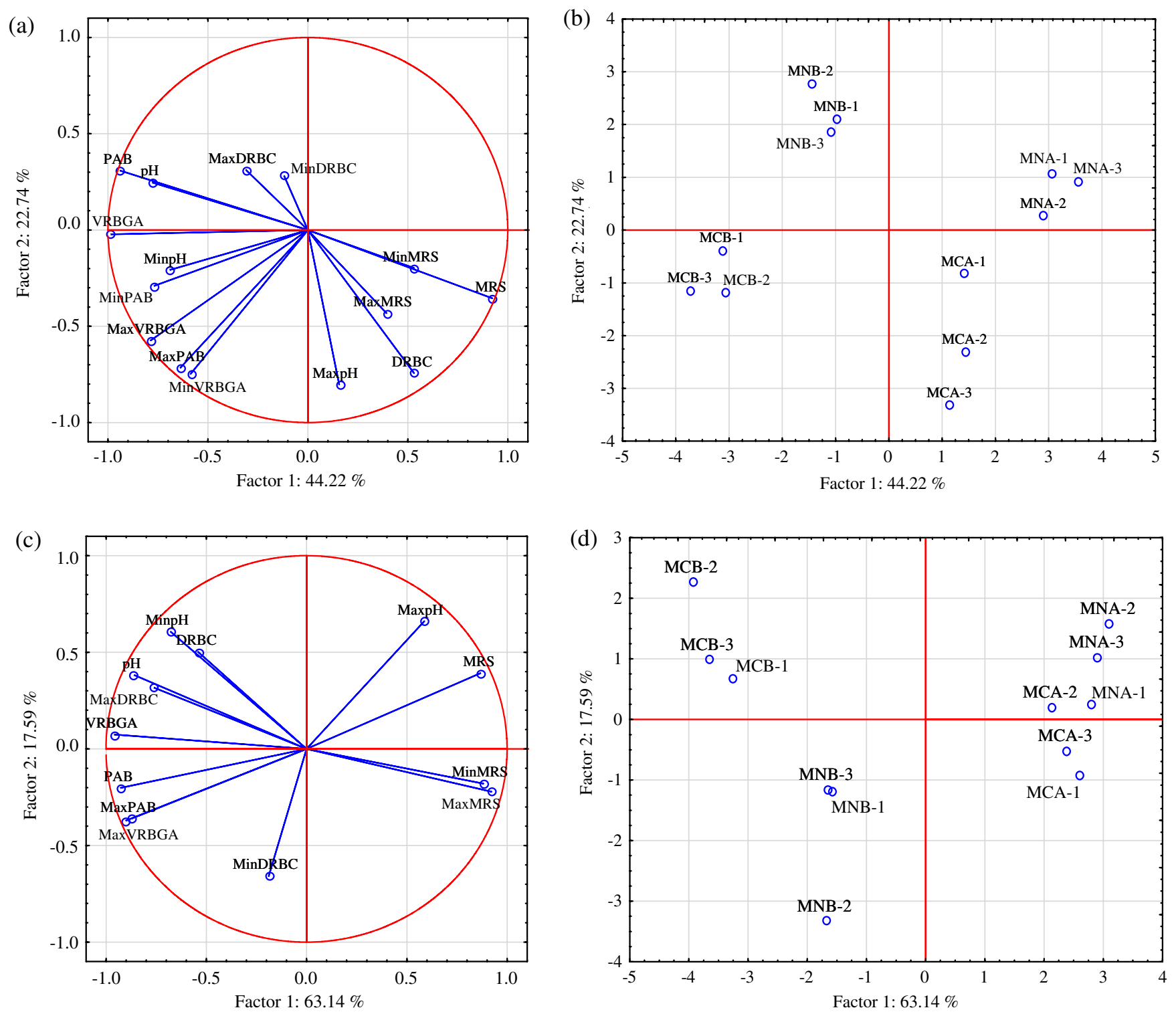

Figure 3. PCA analysis based on the values of microbial and pH changes estimated during the 2012 (a: loading plot; b: score plot) and 2013 (c: loading plot; d: score plot) campaigns. Abbreviations: MRS, Man-Rogosa-Sharpe agar for mesophilic rod LAB; DRBC, dichloran rose bengal chloramphenicol agar for total yeasts and filamentous fungi; VRBGA, violet red bile glucose agar for Enterobacteriaceae; PAB, Pseudomonas agar base for pseudomonads. 'Max' and 'Min' correspond to the maximum and minimum values of $\mathrm{pH}$ and microbial populations detected on the corresponding medium used for counts. The codes (MCA, MCB, MNA and MNA) correspond to the experimental trials and the numbers associated with codes correspond to replicates per trial.

at very low concentrations, both yeasts and $L A B$ could affect the chemical composition, such as $\mathrm{pH}$ and concentration of VOCs, of many foods.

In this regard, high concentrations of acids, such as acetic and nonanoic acids as well as 1-octanol and 2-decenal (E) were found, in this work, in olives inoculated with starter. High concentrations of these compounds indicate alcoholic and heterolactic fermentation. ${ }^{65,66}$ Experimental olives also showed many alcohols at high concentrations; the presence of cis-hexen-1-ol and 1-hexanol in table olives is commonly associated with herbaceous flavours - a pleasant sensory descriptor of many fruit and vegetable fermented foods.

Multivariate data analysis has been widely applied in food processes, ${ }^{67}$ including table olive production. ${ }^{41,43,68,69}$ HCA visibly discriminated trials on the basis of type of fermentation. However, as shown in the dendrograms, low dissimilarity was found among samples collected from the mass of drupes harvested manually or mechanically. The areas and values of both $\mathrm{pH}$ and microbial groups of microorganisms included in the study were proven to be useful variables to discriminate samples. HCA is an unsupervised method that recognizes and distributes data, according to their affinity, in clusters of progressive dissimilarity. In detail, the HCA is a graphical representation of a matrix of distances such as the dendrogram where the objects (trials) are joined together in a hierarchical ascendant analysis from the closest one - i.e. the most similar - to the furthest apart - i.e. the most different.

Since the correlation analysis among variables showed that there were many significant relationships among them, data were subjected to PCA in order to condense the information into a reduced number of factors. Both multivariate statistical approaches (HCA and PCA) showed that the olives produced according to the same type of fermentation were closely related regardless of the type 

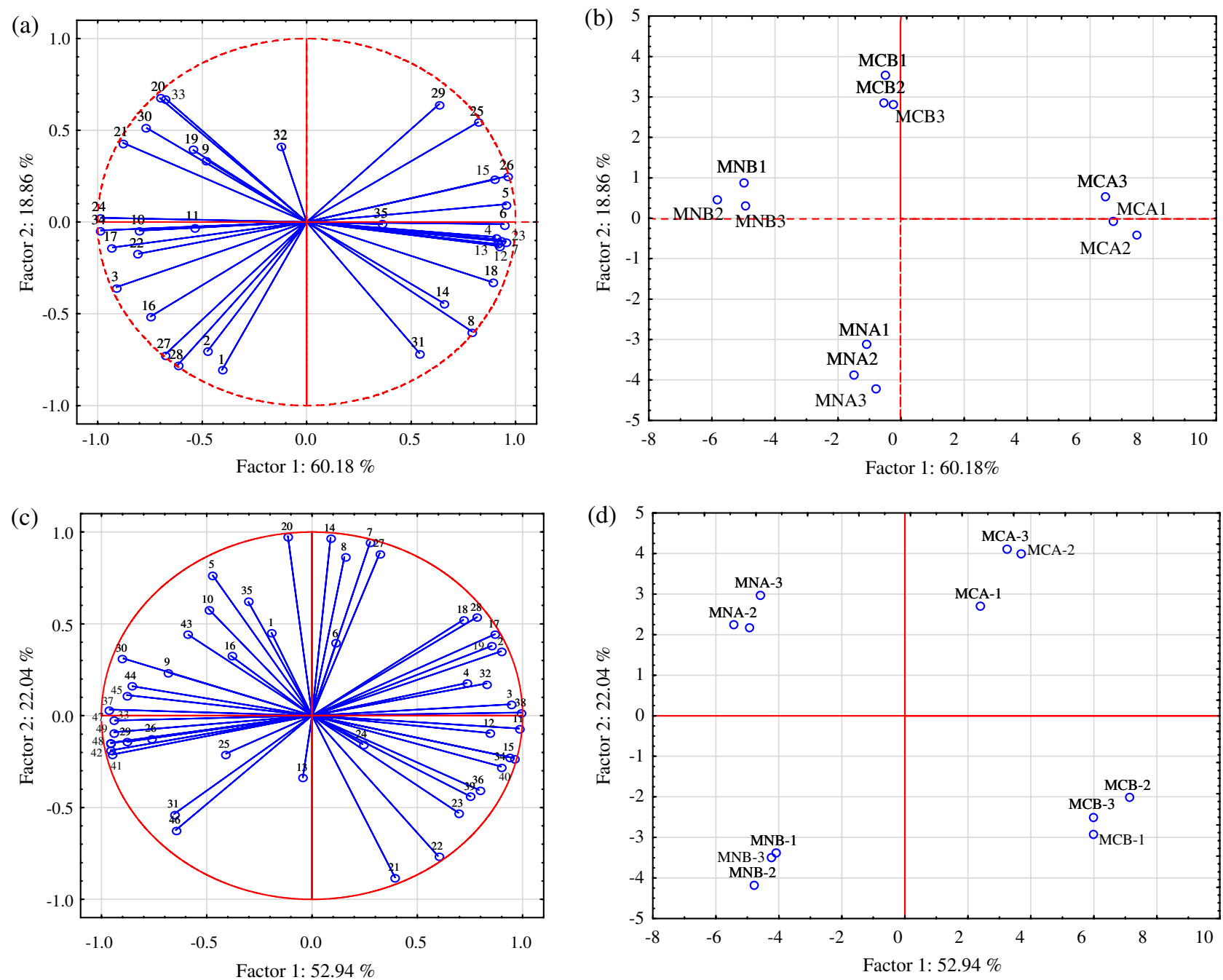

Figure 4. PCA analysis based on the values of VOCs of samples collected at the end (day 210) of olive production in 2012 (a: loading plot; b: score plot) and 2013 (c: loading plot; d: score plot) campaigns. Numbers in the loading plots: 1, acetic acid; 2, butanoic acid; 3, hexadecanoic acid; 4, hexanoic acid; 5, nonanoic acid; 6, octanoic acid; 7, pentanoic acid; 8, propionic acid; 9, 1,4-butanediol; 10, 1-butanol-3-methyl; 11, 1-hexanol; 12, 1-octanol; 13, 2-nonen-1-ol; 14, benzyl alcohol; 15, cis-hexen-1-ol; 16, phenylethyl alcohol; 17, 2-butenal-2-methyl; 18, 2-decenal (E); 19, benzaldehyde; 20, benzaldehyde-2.5-dimethyl; 21, benzaldehyde-3-ethyl; 22, nonanal; 23, octanal; 24, phenylacetaldehyde; 25, vanillin; 26, 2-nonanone; 27, 3 -hydroxybutanone; 28, cyclopentanone; 29, butyrolactone; 30, guaiacol; 31, homoguaiacol; 32, phenol; 33, squalene; 34, styrene; 35, $\alpha$-cubebene for Fig. 2(a); 1, acetic acid; 2, butanoic acid; 3, hexadecanoic acid; 4, hexanoic acid; 5, nonanoic acid; 6, octanoic acid; 7, pentanoic acid; 8, propionic acid; 9, 2-ethylhexanoic acid; 10, heptanoic acid; 11, 1,4-butanediol; 12, 1-butanol-3-methyl; 13, 1-hexanol; 14, 1-octanol; 15, 2-nonen-1-ol; 16, benzyl alcohol; 17, cis-hexen-1-ol; 18, phenylethyl alcohol; 19, 2-butenal-2-methyl; 20, 2-decenal (E); 21, benzaldehyde; 22, benzaldehyde-2.5-dimethyl; 23, benzaldehyde-3-ethyl; 24, nonanal; 25, octanal; 26, phenylacetaldehyde; 27, vanillin; 28, 2-nonanone; 29, 3-hydroxybutanone; 30, cyclopentanone; 31, 4-ethylacetophenone; 32, butyrolactone; 33, 4-methyldihydro-2-(3H)-furanone; 34, guaiacol; 35, homoguaiacol; 36, phenol; 37, 4-ethylphenol; 38, squalene; 39, styrene; $40, \alpha$-cubebene; 41 , benzyl acetate; 42 , cis-3-hexenylacetate; 43 , ethyl dihydrocinnamate; 44 , ethyl lactate; 45 , methyl hexadecanoate; 46, methyl hydrocinnamate; 47 , methyl salicylate; 48, octyl acetate; 49, $\alpha$-terpineol for the Fig. 2(c). Abbreviations: the codes (MCA, MCB, MNA and MNA) correspond to the experimental trials and the numbers associated with codes correspond to replicates per each trial.

of harvesting. Differences in terms of VOC composition estimated among trials might be explained on the basis of variability at strain level found within the L. pentosus populations. On the whole, results found by PCA were in agreement with those obtained by clustering analysis based on $\mathrm{pH}$ and microbiological data.

\section{CONCLUSIONS}

Our study provides an overview of the microbial ecology and chemical parameters of fermented table olives produced with drupes subjected to mechanical harvesting performed using a trunk shaker equipped with an inverse umbrella. Interestingly, statistical analysis of the data showed that, independent of the year of harvest, the addition of starter ensured a rapid decrease in $\mathrm{pH}$ and high concentrations of LAB. In addition, the experimental protocol reduced the growth of spoilage microorganisms during the entire process. The scores associated with undesired off-odours and off-flavours were superimposable on that estimated for the controls performed with manual harvesting. Thus mechanical harvesting performed using the trunk shaker equipped with an inverse umbrella and the addition of starter cultures represents a valuable alternative to manual harvesting for table olive production.

Further investigations will be carried out on other olive varieties for a more thorough validation of this harvest method for table olive production. 
(a)

Biplot (axes F1 and F2: $81.18 \%$ )

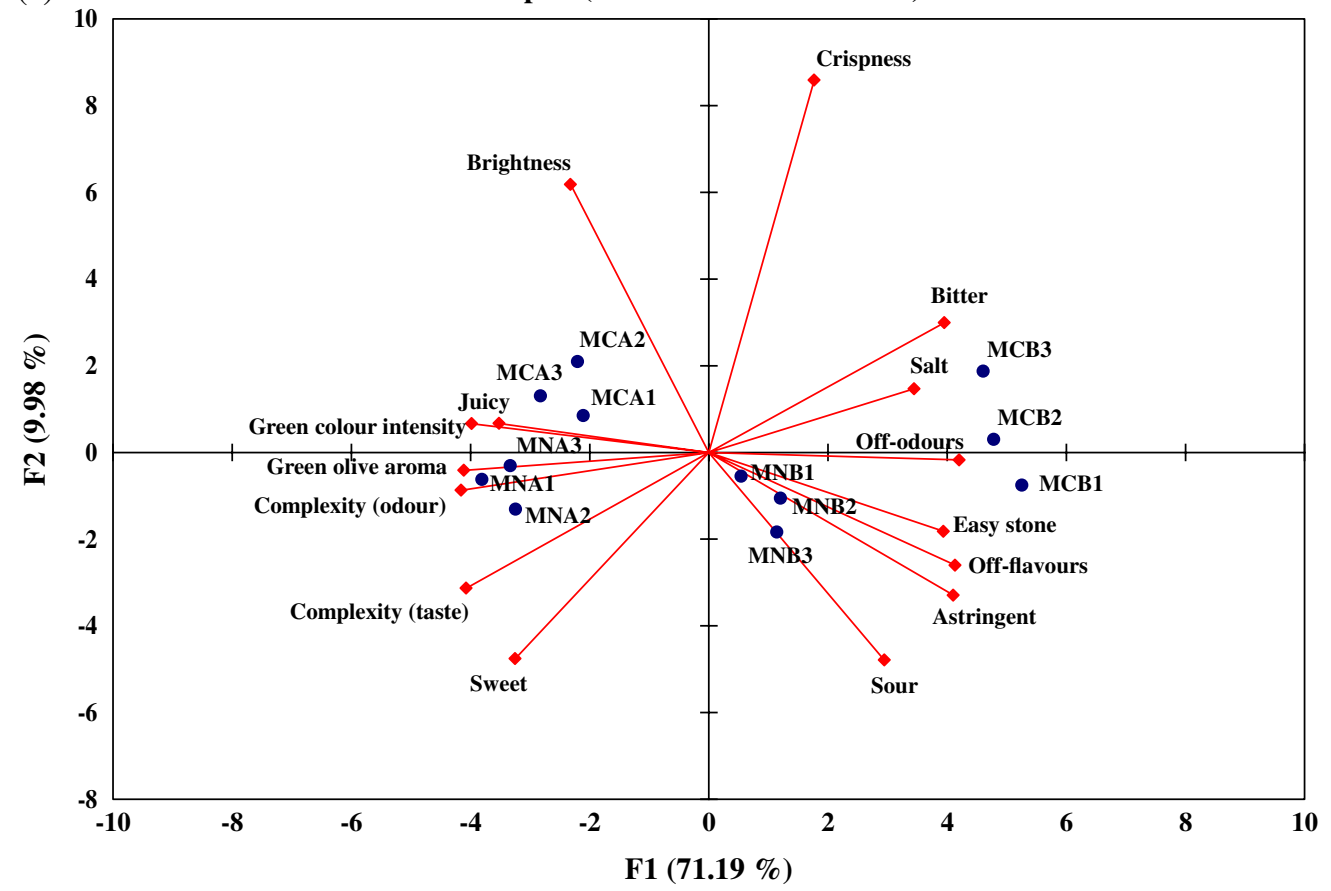

(b)

Biplot (axes F1 and F2: $84.37 \%$ )

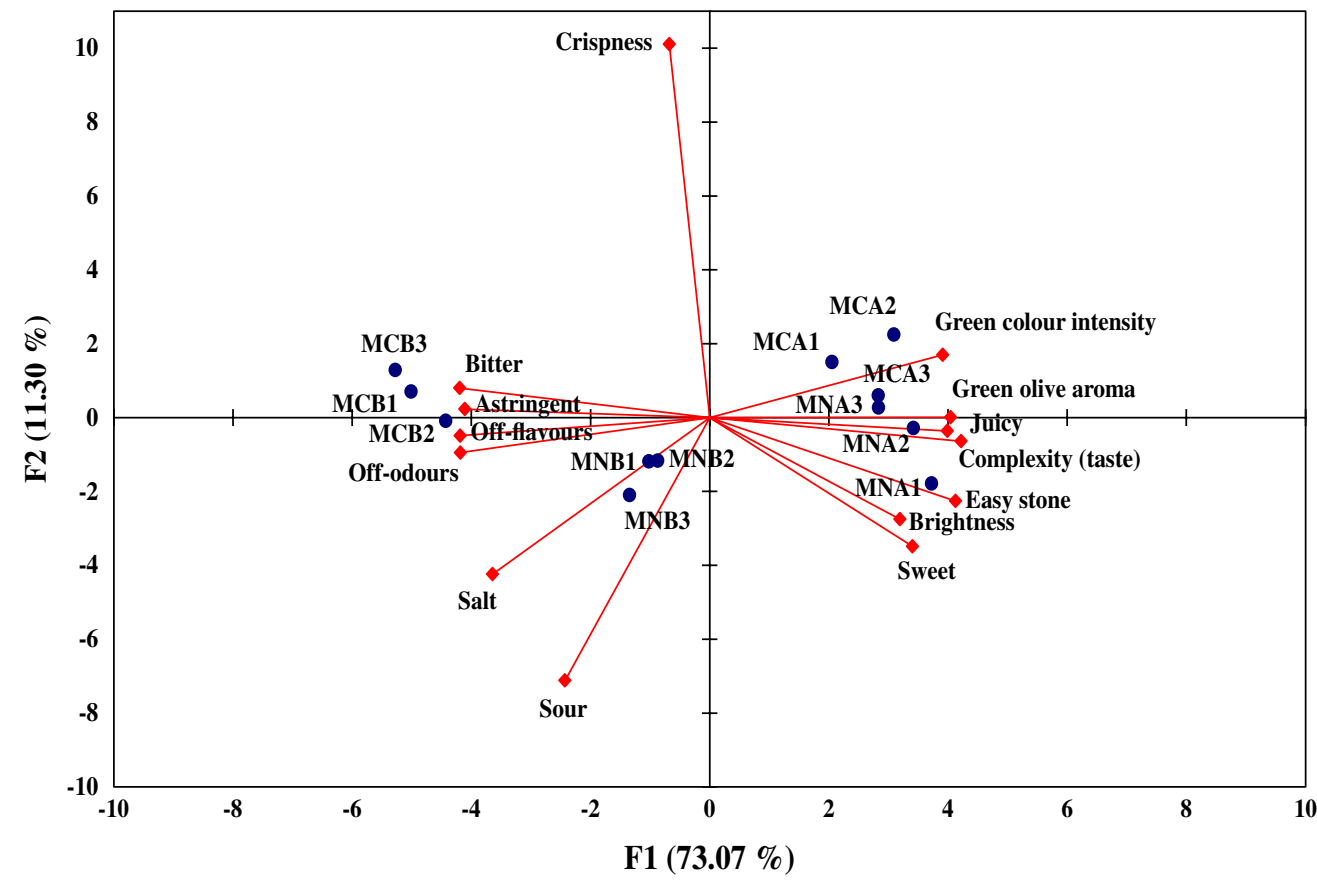

Figure 5. PCA for sensory data of olives at the end of the process (day 210) during the 2012 (a) and 2013 (b) campaigns. Biplot graphs show relationships among factors, variables and treatments. Abbreviations: the codes (MCA, MCB, MNA and MNA) correspond to the experimental trials and the numbers associated with codes correspond to replicates per each trial.

\section{ACKNOWLEDGEMENTS}

This work was financially supported by PON Ricerca e Competitività 2007 - 2013_Progetto “Di.Me.Sa." - Valorizzazione di prodotti tipici della Dieta Mediterranea e loro impiego a fini salu-tistici e nutraceutici. Code of Project: PON02_00667 e PON02_00451_ 3361785. The authors wish to thank the enterprise Crescimanno Pierluigi (Azienda Agricola Angela Consiglio, Castelvetrano, TP, Italy) for its technical support during the harvesting of drupes, the company LALLEMAND Inc. (1620 rue Prefontaine, Montreal Quebec H1W 2 N8, Canada) for its significant support in lyophilizing $L$. pentosus strains, and Antonino Leone and Cinzia Maltese for their support in microbiological and chemical analyses.

\section{SUPPORTING INFORMATION}

Supporting information may be found in the online version of this article. 


\section{REFERENCES}

1 Burns JK, Ferguson L, Glozer K, Krueger WH and Rosecrance RC, Screening fruit loosening agents for black ripe processed table olives. HortScience 43:1449-1453 (2008).

2 Ferguson L, Klonsky K and Martin GC, The olive harvest, in Olive Production Manual, ed. by Sibbett GS, Ferguson L, Coviello JL and Lindstrand M. University of California Agricultural and Natural Resources Publication 3353, pp. 135-140 (2005).

3 Mohsenin NN, Physical Properties of Plant and Animal Materials: Structure, Physical Characteristics and Mechanical Properties. Gordon \& Breach, New York (1986).

4 Van Linden V, Scheerlinck N, Desmet M and De Baerdemaeker J, Factors that affect tomato bruise development as a result of mechanical impact. Postharvest Biol Technol 42:260-270 (2006).

5 Zipori I, Dag A, Tugendhaft $Y$ and Birger R, Mechanical harvesting of table olives: harvest efficiency and fruit quality. HortScience 49:55-58 (2014).

6 Jiménez-Jiménez F, Castro-García S, Blanco-Roldán GL, Ferguson L, Rosa UA and Gil-Ribes JA, Table olive cultivar susceptibility to impact bruising. Postharvest Biol Technol 86:100-106 (2013).

7 Tous J, Olive production systems and mechanization. Acta Hortic 924:169-184 (2011).

8 Vossen P, Pruning Olive Trees: How to Minimize Alternate Bearing And Improve Production. Newsletter of Olive Oil production and Evaluation, Vol. 2(3) (2007).

9 Ferguson L, Miles J, Rosa U, Castro-Garcia S, Krueger WH, Ficthner EJ et al., Developing Mechanical Harvesting for California Black Ripe Table Olives. West Coast Olive Guide, JCS marketing publication, pp. 10-14 (2012).

10 Vega Macias VA, Navarro Rejano L, Guzman Diaz JP, Navarro Garcia C, Higinio Sanchez A and Diaz Montero JM, Recoleccion mecanizada de la aceituna de 'verdeo'. Agricultura 874:376-379 (2005).

11 Zion B, Bechar A, Regev R, Shamir N, Weissblum A, Zipori Y et al., Mechanical harvest of olives: an operations study. Israel J Plant Sci 59:71-84 (2011).

12 Amirante P, Catalano P, Giametta F, Leone A and Montel GL, Vibration analysis of an olive mechanical harvesting system. Agric Eng Int: CIGR Ejournal IX:manuscript PM 07012 (2007).

13 Ferguson L, Rosa UA, Castro-Garcia S, Lee SM, Guinard JX, Burns J et al., Mechanical harvesting of California table and oil olives. Adv Hortic Sci 24:53-63 (2010).

14 Sessiz A and Özcan MT, Olive removal with pneumatic branch shaker and abscission chemical. J Food Eng 76:148-153 (2006).

15 Gambella F, Dimauro C and Paschino F, Evaluation of fruit damage caused by mechanical harvesting of table olives. Trans ASABE 56:1-6 (2013).

16 Servili M, Settanni L, Veneziani G, Esposto S, Massitti O and Taticchi A, The use of Lactobacillus pentosus $1 \mathrm{MO}$ to shorten the debittering process time of black-table olives (cv. Itrana and Leccino): a pilot-scale application. J Agric Food Chem 54:3869-3875 (2006).

17 Hurtado A, Reguant C, Bordons A and Rozès N, Lactic acid bacteria from fermented table olives. Food Microbiol 31:1 -8 (2012).

18 Tofalo R, Perpetuini G, Schirone M, Suzzi G and Corsetti A, Yeast biota associated to naturally fermented table olives from different Italian cultivars. Int J Food Microbiol 161:203-208 (2013).

19 Catania P, Alleri M, Martorana A, Settanni L, Moschetti G and Vallone M, Investigation of a tunnel pasteurizer for 'Nocellara del Belice' table olives processed according to the 'Castelvetrano method'. Grasas Aceites 65:e049 (2014).

20 Lu Z, Breidt F, Plengvidhya V and Fleming HP, Bacteriophage ecology in commercial sauerkraut fermentations. Appl Environ Microbiol 69:3192-3202 (2003).

21 Tofalo R, Perpetuini G, Schirone M, Ciarrocchi A, Fasoli G, Suzzi G et al., Lactobacillus pentosus dominates spontaneous fermentation of Italian table olives. LWT Food Sci Technol 57:710-717 (2014).

22 Aponte M, Blaiotta G, La Croce F, Mazzaglia A, Farina V, Settanni L et al., Use of selected autochthonous lactic acid bacteria for Spanish-style table olive fermentation. Food Microbiol 30:8-16 (2012).

23 Peres C, Catulo L, Brito D and Pintado C, Lactobacillus pentosus DSM 16366 starter added to brine as freeze-dried and as culture in the nutritive media for Spanish style green olive production. Grasas Aceites 59:234-238 (2008).

24 Sabatini N, Mucciarella MR and Marsilio V, Volatile compounds in uninoculated and inoculated table olives with Lactobacillus plantarum (Olea europaea L., cv. Moresca and Kalamata). LWT - Food Sci Technol 41:2017-2022 (2008).
25 Aponte M, Ventorino V, Blaiotta G, Volpe G, Farina V, Avellone G et al., Study of green Sicilian table olive fermentations through microbiological, chemical and sensory analyses. Food Microbiol 27:162-170 (2010).

26 Bautista-Gallego J, Arroyo-López FN, Rantsiou K, Jiménez-Díaz R, Garrido-Fernández A and Cocolin L, Screening of lactic acid bacteria isolated from fermented table olives with probiotic potential. Food Res Int 50:135-142 (2013).

27 Di Cagno R, Coda R, De Angelis M and Gobbetti M, Exploitation of vegetables and fruits through lactic acid fermentation. Food Microbiol 33:1 - 10 (2013).

28 IOC, International Olive Council (2013). [Online]. Available: www.inter nationaloliveoil.org/estaticos/view/132-world-table-olive-figures [30 June 2015].

29 Poiana $\mathrm{M}$ and Romeo FV, Changes in chemical and microbiological parameters of some varieties of Sicily olives during natural fermentation. Grasas Aceites 57:402-408 (2006).

30 Garrido Fernández A, Fernández Díaz MJ and Adams RM, Table Olives: Production and Processing. Chapman \& Hall, London (1997).

31 Martorana A, Alfonzo A, Settanni L, Corona O, La Croce F, Caruso T et al., An innovative method to produce green table olives based on 'pied de cuve' technology. Food Microbiol 50:126-140 (2015).

32 Settanni L, Di Grigoli A, Tornambé G, Bellina V, Francesca N, Moschetti $\mathrm{G}$ et al., Persistence of wild Streptococcus thermophilus strains on wooden vat and during the manufacture of a traditional Caciocavallo type cheese. Int J Food Microbiol 155:73-81(2012a).

33 Torriani S, Felis GE and Dellaglio F, Differentiation of Lactobacillus plantarum, L. pentosus and L. paraplantarum by recA gene sequence analysis and multiplex PCR assay with recA gene-derived primers. Appl Environ Microbiol 67:3450-3454 (2001).

34 Weisburg W, Barns SM, Pelletier DA and Lane DJ, 16S ribosomal DNA amplification for phylogenetic study. J Bacteriol 173:697-703 (1991).

35 Esteve-Zarzoso B, Belloch C, Uruburu F and Querol A, Identification of yeasts by RFLP analysis of the 5.8S rRNA gene and the two ribosomal internal transcribed spacers. Int J Syst Bacteriol 49:329-337 (1999).

36 Settanni L, Sannino C, Francesca N, Guarcello R and Moschetti G, Yeast ecology of vineyards within Marsala wine area (western Sicily) in two consecutive vintages and selection of autochthonous Saccharomyces cerevisiae strains. J Biosci Bioeng 114:606-614 (2012).

37 Pawliszyn J, Applications of Solid Phase Micro-extraction. Royal Society of Chemistry, Cambridge, UK (1999).

38 Corona $\mathrm{O}$, Wine-making with protection of must against oxidation in a warm, semi-arid terroir. S Afr J Enol Vitic 31:58-63 (2010).

39 UNI 10957, Sensory Analysis: Method for Establishing a Sensory Profile in Foodstuffs and Beverages. Standards Library, HIS Engineering 360 (2003).

40 Todeschini R, Introduzione alla Chemiometria. EdiSES, Naples (1998).

41 Bautista-Gallego J, Arroyo-López FN, Romero Gil V, Rodríguez-Gómez F, García-García P and Garrido Fernández A, Chloride salt mixtures affect Gordal cv. green Spanish-style table olive fermentation. Food Microbiol 28:1316-1325 (2011).

42 Blana VA, Grounta A, Tassou CC, Nychas GJE and Panagou EZ, Inoculated fermentation of green olives with potential probiotic Lactobacillus pentosus and Lactobacillus plantarum starter cultures isolated from industrially fermented olives. Food Microbiol 38:208-218 (2014).

43 Rodríguez-Gómez F, Bautista-Gallego J, Romero-Gil V, Arroyo-López FN, Garrido-Fernández A and García P, Effects of salt mixtures on Spanish green table olive fermentation performance. LWT - Food Sci Technol 46:56-63 (2012).

44 Rodríguez-Gómez F, López-López A, Romero-Gil V, Arroyo-López FN, Moreno-Baquero JM, Garrido-Fernández A etal., Effect of post-fermentation storage on Spanish-style green Manzanilla olives. LWT - Food Sci Technol 57:789-793 (2014).

45 Jolliffe IT, Principal Component Analysis. Springer, New York (1986).

46 Dillon WR and Goldstein M, Multivariate Analysis: Methods and Applications. Wiley, New York (1984).

47 Mazzei P, Francesca N, Moschetti G and Piccolo A, NMR spectroscopy evaluation of direct relationship between soils and molecular composition of red wines from Aglianico grapes. Anal Chim Acta 673:167-172 (2010).

48 Clavijo A, Calderon IL and Paneque P, Yeast assessment during alcoholic fermentation inoculated with a natural 'pied de cuve' or a commercial yeast strain. World J Microb Biot 27:1569-1577 (2011). 
49 Li E, Liu C and Liu Y, Evaluation of yeast diversity during wine fermentations with direct inoculation and pied de cuve method at an industrial scale. J Microbiol Biotechnol 22:960-966 (2012).

50 Ubeda Iranzo JF, Gonzalez Magana F and Gonzalez Vinas MA, Evaluation of the formation of volatiles and sensory characteristics in the industrial production of white wines using different commercial strains of the genus Saccharomyces. Food Control 11:143-147 (2000).

51 Oblinger JL, Kennedy JE and Langston DM, Microflora recovered from foods on Violet Red Bile Agar with and without glucose and incubated at different temperatures. J Food Protect 45:948-952 (1982).

52 Silvestri V, Francesca N, Settanni L and Moschetti G, Attitudini tecnologiche di batteri lattici starter per la fermentazione di olive verdi da mensa. Ind Aliment - Italy XLVIII:44-55 (2009).

53 Biavati B and Sorlini C, Tassonomia microbica, in Microbiologia generale e agraria, ed. by Biavati B and Sorlini C. Casa Editrice Ambrosiana, Milan, pp. 273-322 (2007).

54 Galli Volonterio A, Famiglie e generi microbici di interesse alimentare, in Microbiologia degli alimenti, ed. by Galli Volonterio A. Casa Editrice Ambrosiana, Milan, pp. 120-144 (2005).

55 Arroyo-López FN, Querol A, Bautista-Gallego J and Garrido-Fernández A, Role of yeasts in table olive production. Int J Food Microbiol 128:189-196 (2008).

56 Bleve G, Tufariello M, Durante M, Grieco F, Ramires FA, Mita G et al., Physico-chemical characterization of natural fermentation process of Conservolea and Kalamata table olives and development of a protocol for the pre-selection of fermentation starters. Food Microbiol 46:368-382 (2015).

57 Nout MJR and Rombouts FM, Fermented and acidified plant foods, in The Microbiological Safety and Quality of Food, ed. by Lund BM, Baird-Parker TC and Gould GW. Aspen, Frederick, MD, pp. 685-737 (2000).

58 Romero-Gil V, Bautista-Gallego J, Rodríguez-Gómez F, García-García P, Jiménez-Díaz R, Garrido-Fernández A et al., Evaluating the individual effects of temperature and salt on table olive related microorganisms. Food Microbiol 33:178-184 (2013).

59 Tofalo R, Schirone M, Perpetuini G, Angelozzi G, Suzzi G and Corsetti A, Microbiological and chemical profiles of naturally fermented table olives and brines from different Italian cultivars. Antonie Van Leeuwenhoek 102:121-131 (2012).

60 Tofalo R, Schirone M, Perpetuini G, Suzzi G and Corsetti A, Development and application of a real-time PCR-based assay to enumerate total yeasts and Pichia anomala, Pichia guillermondii and Pichia kluyveri in fermented table olives. Food Control 23:356-362 (2012).

61 Panagou EZ, Tassou CC and Katsaboxakis CZ, Induced lactic acid fermentation of untreated green olives of the Conservolea cultivar by Lactobacillus pentosus. J Sci Food Agric 83:667-674 (2003).

62 Fernández-Díez MJ, de Castro R, Garrido-Fernández A, González F, González-Pellissó F, Nosti M et al., Biotecnologia de la Aceitunas de Mesa. CSIC, Madrid (1985).

63 Sannino C, Francesca N, Corona C, Settanni L, Cruciata M and Moschetti $\mathrm{G}$, Effect of the natural winemaking process applied at industrial level on the microbiological and chemical characteristics of wine. $J$ Biosci Bioeng 116:347-356 (2013).

64 Francesca N, Romano R, Sannino C, Le Grottaglie L, Settanni L and Moschetti G, Evolution of microbiological and chemical parameters during red wine making with extended post-fermentation maceration. Int J Food Microbiol 171:84-93 (2014).

65 Sabatini N, Perri E and Marsilio V, An investigation on molecular partition of aroma compounds in fruit matrix and brine medium of fermented table olives. Innov Food Sci Emerg 10:621 -626 (2009).

66 Bleve G, Tufariello M, Durante M, Perbellini E, Ramires FA, Grieco $\mathrm{F}$ et al., Physico-chemical and microbiological characterization of spontaneous fermentation of Cellina di Nardò and Leccino table olives. Front Microbiol 5:1 - 18 (2014).

67 Berrueta LA, Alonso-Salces RM and Héberger K, Supervised pattern recognition in food analysis. J Chromatogr A 1158:196-214 (2007).

68 Rodríguez-Gómez F, Romero-Gil V, Bautista-Gallego J and GarridoFernández A, Multivariate analysis to discriminate yeasts strains technological applications in table olive processing. World J Microb Biot 28:1761-1770 (2012).

69 Rodríguez-Gómez F, Bautista-Gallego J, Arroyo-López FN, Romero-Gil $\mathrm{V}$, Jiménez-Díaz $\mathrm{R}$ and Garrido-Fernández A, Table olive fermentation with multifunctional Lactobacillus pentosus strains. Food Control 34:96-105 (2013). 